\title{
Computing gravitational waves from slightly nonspherical stellar collapse to a black hole: Odd-parity perturbation
}

\author{
Tomohiro Harada ${ }^{1 *}$, Hideo Iguchi ${ }^{2 \dagger}$, and Masaru Shibata ${ }^{3 \ddagger}$ \\ ${ }^{1}$ Department of Physics, Waseda University, Shinjuku, Tokyo 169-8555, Japan \\ ${ }^{2}$ Department of Physics, Tokyo Institute of Technology, Meguro, Tokyo 152-8550, Japan \\ ${ }^{3}$ Department of Earth Science and Astronomy, Graduate School of Arts and Sciences, \\ University of Tokyo, Meguro, Tokyo 153-8902, Japan
}

(Dated: November 16, 2018)

\begin{abstract}
Nonspherical stellar collapse to a black hole is one of the most promising gravitational wave sources for gravitational wave detectors. We numerically study gravitational waves from a slightly nonspherical stellar collapse to a black hole in linearized Einstein theory. We adopt a spherically collapsing star as the zeroth-order solution and gravitational waves are computed using perturbation theory on the spherical background. In this paper, we focus on the perturbation of odd-parity modes. Using the polytropic equations of state with polytropic indices $n_{p}=1$ and 3 , we qualitatively study gravitational waves emitted during the collapse of neutron stars and supermassive stars to black holes from a marginally stable equilibrium configuration. Since the matter perturbation profiles can be chosen arbitrarily, we provide a few types for them. For $n_{p}=1$, the gravitational waveforms are mainly characterized by a black hole quasinormal mode ringing, irrespective of perturbation profiles given initially. However, for $n_{p}=3$, the waveforms depend strongly on the initial perturbation profiles. In other words, the gravitational waveforms strongly depend on the stellar configuration and, in turn, on the ad hoc choice of the functional form of the perturbation in the case of supermassive stars.
\end{abstract}

PACS numbers: 04.30.Db, 04.25.Dm, 04.30.Nk, 04.70.Bw

\section{INTRODUCTION}

Detection of gravitational waves is one of the greatest challenges in experimental and theoretical physics in this decade. Several kilometer-size laser interferometers, such as TAMA [1], the Laser Interferometric Gravitational Wave Observatory (LIGO) [2], and GEO 3] are in operation now and VIRGO [4] will be in several years. In addition to these ground-based detectors, the Laser Interferometer Space Antenna (LISA) with an arm length of $5 \times 10^{6} \mathrm{~km}$ has been proposed 5, 6], and is planned to start taking observations in 2012.

Nonspherical stellar collapse is one of the most promising sources of gravitational waves for both ground-based detectors and space antennas. Ground-based interferometric detectors have a good sensitivity in the frequency range between $\sim 10$ and $1 \mathrm{kHz}$. Thus, the stellar core collapse of a massive star to a neutron star [7] or a black hole [8] is one of the targets. According to [8], at the formation of a massive black hole, quasinormal modes of a black hole are excited and gravitational waves of high amplitude associated with the quasinormal modes are emitted. (See [9] for a review of black hole quasinormal modes.) The frequency of gravitational waves associated with the fundamental quadrupole quasinormal modes of rotating black holes is [10]

$$
f \sim(0.03-0.08) M^{-1} \simeq(300-800)\left(\frac{M}{20 M_{\odot}}\right)^{-1} \mathrm{~Hz}
$$

where the frequency is higher for more rapidly rotating black holes. Equation (1.1) indicates that formation of black holes of mass $\gtrsim 20 M_{\odot}$ may be a promising source for laser-interferometric detectors.

The frequency band of space antennas is between $\sim 10^{-4}$ and $\sim 0.1 \mathrm{~Hz}[5]$. This suggests that the formation of supermassive black holes may be one of the most promising sources. Although the actual scenarios by which supermassive black holes form are still uncertain, viable stellar dynamical and hydrodynamical routes leading to the formation of supermassive black holes have been proposed [11, 12, 13. In typical hydrodynamical scenarios, a supermassive gas cloud is built up from multiple collisions of stars or small gas clouds in stellar clusters to form a supermassive star. Supermassive stars ultimately collapse to black holes following quasistationary cooling and

\footnotetext{
* Electronic address:harada@gravity.phys.waseda.ac.jp

$\dagger$ Electronic address:iguchi@th.phys.titech.ac.jp

$\ddagger$ Electronic address:shibata@provence.c.u-tokyo.ac.jp
} 
contraction to the onset of radial instability 14, 15. Such dynamical formation of supermassive black holes may be a strong gravitational wave source for LISA [16, 17, 18, 19, 20].

The most hopeful approach for the computation of gravitational waves from stellar collapse to a black hole is to numerically solve the fully nonlinear coupled equations of Einstein and the general relativistic hydrodynamic equations. There has been much progress in this field in the last few years 21]. However, it is not technically easy to compute gravitational waves with high precision in numerical relativity, since the amplitude of gravitational waves associated with the stellar collapse is not very large and as a result, they could be contaminated by numerical noises and/or gauge modes. To cross-check the numerical results and also to compute precise gravitational waveforms, it is desirable to have another method.

As an alternative approach, linear perturbation theory has been developed [22, 23, 24]. In this approach, we decompose the fully nonlinear metric and matter field of slightly nonspherical profiles into a spherically symmetric dynamical field and linearized nonspherical perturbations. Because of progress in numerical techniques and computational resources, Einstein's equations in spherically symmetric spacetime can be now accurately computed at low computational cost. Furthermore, due to the spherical symmetry of the background spacetime, the perturbations can be expanded in spherical harmonics. Thus all the equations for gravitational waves reduce to two simple $(1+1)$ wave equations of even and odd parities, which can be numerically solved with high precision. Although this method is applicable only to slightly nonspherical problems, the gravitational waveforms of small amplitude associated with stellar collapse can be computed with high accuracy. The result is also useful for calibration of fully nonlinear numerical results.

The history of progress in linear perturbation theory of a spherically symmetric spacetime is as follows. The first work in this field was done by Regge and Wheeler [25] and Zerilli [26]. They derived the linear perturbation equations of odd parity [25] and of even parity [26] in Schwarzschild background spacetime. Subsequently, a gauge-invariant formalism of linear perturbations was developed by Moncrief [27]. Extending his work, Cunningham, Price, and Moncrief [22] derived perturbation equations on the Oppenheimer-Snyder solution for a collapsing uniform dust ball [28], and computed gravitational waves emitted during the gravitational collapse of a dust ball to a black hole. Gerlach and Sengupta 29] subsequently developed a gauge-invariant formulation of the linear perturbation on general spherically symmetric spacetimes. Using the Gerlach-Sengupta formalism, Seidel and co-workers 23] computed gravitational radiation from stellar core collapses, focusing mainly on the waveforms associated with the formation of neutron stars. They numerically solved the spherically symmetric general relativistic hydrodynamic equations using the May-White scheme [30, 31]. Harada et al. 32] studied scalar gravitational radiation from a collapsing homogeneous dust ball in scalar-tensor theories of gravity, using a similar method to that of Cunningham, Price, and Moncrief [22]. Iguchi, Nakao, and Harada [33, 34] studied nonspherical perturbations of a collapsing inhomogeneous dust ball, which is described by the Lemaître-Tolman-Bondi solution 35. Recently, Gundlach and Martín-García 24 have developed a covariant gauge-invariant formulation of nonspherical perturbations on spherically symmetric spacetimes with a perfect fluid, and derived coordinate-independent matching conditions for perturbations at the stellar surface.

The dynamics of spherically symmetric spacetimes have been often studied using a method developed by May and White [30]. In this scheme, spherically symmetric spacetimes are described in terms of the so-called Misner and Sharp coordinate system [36], in which a spacelike comoving slicing and an orthogonal time coordinate are adopted. This formulation is robust for the simulation of oscillating spherical stars and stellar core collapse to neutron stars. However, it is not robust enough to carry out simulations for black hole formation, because the computation often crashes before all of the matter is swallowed into a black hole due to inappropriate choice of the slicing condition [54].

To compute black hole formation, a null formulation proposed by Hernandez and Misner is well suited [37]. In this formulation, spacetime is foliated by an outgoing null coordinate and thus the whole region outside the black hole horizon can be covered. The singularity avoidance of the null foliation is assured until the foliation reaches an event horizon if the cosmic censorship holds. Using this formulation, Miller and Motta 38 performed the numerical simulation of collapse to black hole formation. Baumgarte, Shapiro, and Teukolsky [39] used this formulation to study neutrino emission in the delayed collapse of hot neutron stars to black holes. This formulation was also applied to the study of cosmic censorship [34, 40] and the formation of primordial black holes [41]. Linke et al. [42] also computed the spherical collapse of supermassive stars using an outgoing null coordinate but different radial coordinate, i.e., Bondi metric to study the neutrino emissivity during the collapse.

In this paper, we present a new implementation in linearized Einstein theory, in which spherical background spacetimes are computed with the Hernandez-Misner scheme, while nonspherical linear perturbations are treated using the single-null coordinate system. With the Hernandez-Misner scheme, it is possible to follow spherical stellar collapse to a black hole until almost all the matter has collapsed below the event horizon. The null coordinate system is well-suited for computation of gravitational waves emitted near the event horizon, which we want to study here. As a related work to the present treatment, Siebel et al. [43] presented simulations of gravitational collapse of neutron stars to black holes and the computation of quasinormal ringing in the spherically symmetric Einstein-fluid-Klein-Gordon system using an outgoing null coordinate without linear approximation. 
This paper is organized as follows. In Sec. II we briefly review the evolution equations for spherically symmetric spacetimes in terms of the Hernandez-Misner null formulation. In Sec. III we describe the evolution equations for odd-parity gauge-invariant perturbations in the single-null coordinate system and then derive the explicit matching conditions at the stellar surface. In Sec. IV we explain the method for computation of gravitational waves in our gauge-invariant formalism. In Sec. $\mathrm{V}$ we describe numerical techniques adopted in the current implementation. In Secs. VI and VII, we present the numerical results of test simulations, and gravitational waveforms from the collapse of a supermassive star and neutron star to black holes, respectively. Section VIII is devoted to a summary. We adopt geometrical units in which $G=c=1$, where $G$ and $c$ denote the gravitational constant and speed of light, respectively.

\section{BACKGROUND SPACETIME}

\section{A. $2+2$ split of spherically symmetric spacetimes}

We decompose a spherically symmetric spacetime $\mathcal{M}$ into a product as $\mathcal{M}=\mathcal{M}^{2} \times \mathcal{S}^{2}$. Namely a metric is written as

$$
g_{\mu \nu} \equiv \operatorname{diag}\left(g_{A B}, R^{2} \gamma_{a b}\right)
$$

where $g_{A B}, R$, and $\gamma_{a b}$ are the $(1+1)$ Lorentz metric, scalar function on $\mathcal{M}^{2}$, and unit curvature metric on $\mathcal{S}^{2}$. The greek indices $\mu, \nu, \ldots$, capital latin indices $A, B, \ldots$, and small Latin indices $a, b, \ldots$ denote the spacetime components, $\mathcal{M}^{2}$ components and $\mathcal{S}^{2}$ components, respectively. The covariant derivatives on $\mathcal{M}, \mathcal{M}^{2}$, and $\mathcal{S}^{2}$ are denoted as $; \mu$, $\mid A$ and $: a$, i.e., we define them from the conditions $g_{\mu \nu ; \lambda}=0, g_{A B \mid C}=0$, and $\gamma_{a b: c}=0$.

The stress-energy tensor for general spherically symmetric spacetimes is given by

$$
t_{\mu \nu}=\operatorname{diag}\left(t_{A B},\left(t_{a}^{a} / 2\right) R^{2} \gamma_{a b}\right)
$$

The totally antisymmetric covariant unit tensors $\epsilon_{A B}$ on $\mathcal{M}^{2}$ and $\epsilon_{a b}$ on $\mathcal{S}^{2}$ are defined as

$$
\epsilon_{A C} \epsilon^{C B}=-g_{A}^{B}, \quad \epsilon_{a c} \epsilon^{c b}=\gamma_{a}^{b} .
$$

\section{B. Hernandez-Misner formulation of general relativistic hydrodynamics}

We choose the Hernandez-Misner coordinate system of the form

$$
d s^{2}=-e^{2 \psi} d u^{2}-2 e^{\psi+\lambda / 2} d u d x+R^{2}\left(d \theta^{2}+\sin ^{2} \theta d \phi^{2}\right),
$$

where $x$ is a comoving coordinate, and $\psi, \lambda$, and $R$ are functions of $u$ and $x$.

We assume that the stars are composed of a perfect fluid, for which the energy-momentum tensor is written as

$$
t_{\mu \nu}=(\epsilon+p) u_{\mu} u_{\nu}+p g_{\mu \nu}
$$

where $\epsilon, p$, and $u^{\mu}$ are the energy density, pressure and four velocity of the fluid.

We define the following new variables

$$
\begin{aligned}
U & \equiv e^{-\psi} R_{, u}, \\
\Gamma & \equiv e^{-\lambda / 2} R_{, x}-U=\sqrt{1-\frac{2 m}{R}+U^{2}}
\end{aligned}
$$


where $m$ is the Misner-Sharp quasilocal mass [36]. Then, the field equations are written in the form [37]

$$
\begin{aligned}
U_{, u}= & -\frac{e^{\psi}}{1-c_{s}^{2}}\left[\frac{\Gamma e^{-\lambda / 2}}{\epsilon+p} p_{, x}+\frac{m+4 \pi R^{3} p}{R^{2}}\right] \\
& -\frac{e^{\psi} c_{s}^{2}}{1-c_{s}^{2}}\left(e^{-\lambda / 2} U_{, x}+\frac{2 U \Gamma}{R}\right), \\
R_{, u}= & e^{\psi} U \\
m_{, u}= & -e^{\psi} 4 \pi R^{2} p U, \\
e^{-\lambda / 2}= & \frac{\Gamma+U}{R_{, x}}, \\
n= & \frac{e^{-\lambda / 2}}{4 \pi R^{2}} f, \\
\left(\frac{\epsilon}{n}\right)_{, u}= & -p\left(\frac{1}{n}\right), \\
m_{, x}= & 4 \pi R^{2} e^{\lambda / 2}(\epsilon \Gamma-p U), \\
\psi_{, x}= & \frac{1}{\Gamma} U_{, x}+e^{\lambda / 2} \frac{m+4 \pi p R^{3}}{\Gamma R^{2}} .
\end{aligned}
$$

$n$ is the baryon rest-mass density, $f=f(x)$ is an arbitrary function associated with the rescaling of the radial coordinate, and $c_{s}$ is the sound speed which is defined by

$$
c_{s}^{2} \equiv\left(\frac{\partial p}{\partial \epsilon}\right)_{s=\mathrm{const}}
$$

where $s$ denotes the entropy.

The regularity condition at $x=0$ gives the boundary conditions as

$$
\begin{aligned}
R & =0 \\
U & =0 \\
\Gamma & =1 \\
m & =0
\end{aligned}
$$

The boundary condition at the stellar surface $x=x_{\mathrm{s}}$ is given by

$$
p=0
$$

In the original form of the Hernandez-Misner formalism [37], $f$ is chosen to be unity. In this case, $x$ coincides with the conserved mass $\mu$ contained in the interior to a shell. Another candidate for $f$ is

$$
f(x)=\frac{4 \pi n\left(u_{0}, x\right) x^{2}}{(\Gamma+U)\left(u_{0}, x\right)}
$$

In this case, $x$ coincides with the circumferential radius of the shell on the null surface $u=u_{0}$. In this paper we adopt Eq. (2.22) for $f$, since it has a nicer feature for the integration of nonspherical perturbations which we will describe in the next section.

We assume that the exterior of the star is a vacuum. Then, the zeroth-order solution is the Schwarzschild spacetime as

$$
d s^{2}=-\left(1-\frac{2 M}{R}\right) d T^{2}+\left(1-\frac{2 M}{R}\right)^{-1} d R^{2}+R^{2}\left(d \theta^{2}+\sin ^{2} \theta d \phi^{2}\right),
$$

where $M$ is the gravitational mass of the system. To compute gravitational waves in this background, it is convenient to introduce null coordinates $\bar{u}$ and $\bar{v}$ defined as

$$
\begin{aligned}
& \bar{u}=T-R_{*}, \\
& \bar{v}=T+R_{*},
\end{aligned}
$$


where

$$
R_{*} \equiv R+2 M \ln \left(\frac{R}{2 M}-1\right)
$$

Then, the line element is of the form

$$
d s^{2}=-\left(1-\frac{2 M}{R}\right) d \bar{u} d \bar{v}+R^{2}\left(d \theta^{2}+\sin ^{2} \theta d \phi^{2}\right) .
$$

In the following, we refer to the standard outgoing null coordinate $\bar{u}$ as an observer time coordinate according to [39].

The ratio of the proper time interval $d \tau_{\mathrm{s}}$ of an observer at the stellar surface to the observer time interval $d \bar{u}$ is given by

$$
\frac{d \tau_{\mathrm{s}}}{d \bar{u}}=(\Gamma+U)\left(u, x_{\mathrm{s}}\right)
$$

The lapse function $\alpha$ at $x$ is defined by the ratio of the proper time interval $d \tau$ of each fluid element to the observer time interval $d \bar{u}$ as

$$
\alpha(u, x) \equiv \frac{d \tau}{d \bar{u}}=\frac{d \tau}{d \tau_{\mathrm{s}}} \frac{d \tau_{\mathrm{s}}}{d \bar{u}}=\frac{e^{\psi(u, x)}}{e^{\psi\left(u, x_{\mathrm{s}}\right)}}(\Gamma+U)\left(u, x_{\mathrm{s}}\right) .
$$

We note that the lapse function $\alpha$ is directly related to the observed redshift $z$ as $\alpha=1 /(1+z)$.

In solving the dynamics of a spherical star, the boundary condition for $\psi$ is arbitrary. In the present computation, we choose $e^{\psi}\left(u, x_{\mathrm{s}}\right)=1$ for the boundary condition of $\psi$. Then, we can identify the null coordinate $u$ with the proper time $\tau_{\mathrm{s}}$ of the comoving observer at the stellar surface.

\section{ODD-PARITY PERTURBATIONS}

\section{A. Gauge-invariant perturbations}

Perturbed metric and matter fields of odd parity are denoted by

$$
\begin{aligned}
\Delta g_{\mu \nu} & =\left(\begin{array}{cc}
0 & h_{A} S_{a} \\
h_{A} S_{a} & h\left(S_{a: b}+S_{b: a}\right)
\end{array}\right), \\
\Delta t_{\mu \nu} & =\left(\begin{array}{cc}
0 & \Delta t_{A} S_{a} \\
\Delta t_{A} S_{a} & \Delta t\left(S_{a: b}+S_{b: a}\right)
\end{array}\right),
\end{aligned}
$$

where $Y, S_{a} \equiv \epsilon_{a}{ }^{b} Y_{: b}$ and $S_{a: b}+S_{b: a}$ are the scalar, vector and tensor harmonics, respectively. Here, the suffices $l$ and $m$ are omitted for simplicity. The scalar harmonic function $Y$ satisfies

$$
\gamma^{a b} Y_{: a b}=-l(l+1) Y .
$$

The gauge-invariant perturbations are defined as

$$
\begin{aligned}
k_{A} & \equiv h_{A}-h_{\mid A}+2 h v_{A}, \\
L_{A} & \equiv \Delta t_{A}-Q h_{A}, \\
L & \equiv \Delta t-Q h,
\end{aligned}
$$

where

$$
v_{A} \equiv \frac{R_{\mid A}}{R}
$$

$L$ is identically zero for $l=1$, and no perturbation of odd parity appears for $l=0$.

For a perfect fluid, the matter perturbations of odd parity are only specified by the four-velocity perturbations as

$$
\Delta u_{\mu}=\left(0, \beta S_{a}\right),
$$

where $\beta$ is a function of $x$ and $u$ and completely determines the matter perturbations. The concrete form is determined by solving the field equations [cf. Eq. [3.10] ]. In terms of $\beta$, the gauge-invariant perturbations are written as

$$
L_{A}=\beta(\epsilon+p) u_{A},
$$

and $L=0$. 


\section{B. Field equations: interior}

The covariant field equations for nonspherical perturbations in the stellar interior were derived by Gerlach and Sengupta [29] for general matter fields and have been recently reformulated by Gundlach and Martín-García 24] for a perfect fluid. We follow 24] to derive the basic equations for the perturbations.

The perturbation equation for the matter field, $\Delta\left(t_{\nu ; \mu}^{\mu}\right)=0$, is integrated to give

$$
\beta=-\frac{e^{-\lambda / 2}}{R^{2}(\epsilon+p)} j,
$$

where $j=j(x)$ is an arbitrary function of $x$. Integration of $j$ by $d x$ yields a conserved quantity $J$ as

$$
J=\int j d x
$$

where $\oint J S_{\phi} \sin \theta d \theta d \phi$ corresponds to the $z$ component of angular momentum for $l=1$.

At $R=0, \beta$ should satisfy the regularity condition as

$$
\beta=R^{l+1} \bar{\beta},
$$

where $\bar{\beta}$ is a regular function at $R=0$. The regularity of $\beta$ also leads to the following condition

$$
e^{-\lambda / 2} j=R^{l+3} \bar{j}
$$

where $\bar{j}$ is a regular function at $R=0$. In terms of the function $j$, the gauge-invariant matter perturbations are described as

$$
\begin{aligned}
L_{u} & =\frac{e^{\psi-\lambda / 2}}{R^{2}} j, \\
L_{x} & =\frac{1}{R^{2}} j .
\end{aligned}
$$

Thus, in the following, we specify $j$ instead of $\beta$.

The metric perturbations of odd parity are characterized by a master variable as

$$
\Pi \equiv e^{-\psi-\lambda / 2}\left[\left(\frac{k_{x}}{R^{2}}\right)_{, u}-\left(\frac{k_{u}}{R^{2}}\right)_{, x}\right],
$$

where $\Pi$ is related to a variable $\tilde{\pi}_{1}$ introduced in $[22]$ as $\tilde{\pi}_{1}=l(l+1) R^{2} \Pi$. $\Pi$ should satisfy the regularity condition at $R=0$ as

$$
\Pi=R^{l-2} \bar{\Pi}
$$

where $\bar{\Pi}$ is a regular function at $R=0$. $\bar{\Pi}$ satisfies the following wave equation for $l \geq 2$

$$
\begin{aligned}
- & 2 e^{-\psi-\lambda / 2} \bar{\Pi}_{, x u}+e^{-\psi-\lambda / 2}\left(e^{\psi-\lambda / 2} \bar{\Pi}_{, x}\right)_{, x} \\
+ & \frac{2(l+1)}{R}\left\{-(\Gamma+U) e^{-\psi} \bar{\Pi}_{, u}+\Gamma e^{-\lambda / 2} \bar{\Pi}_{, x}\right\} \\
& -(l+2)\left[4 \pi(\epsilon-p)+(l-2) \frac{2 m}{R^{3}}\right] \bar{\Pi} \\
=16 \pi & {\left[R e^{-\psi-\lambda / 2}\left(e^{\psi} \bar{j}\right)_{, x}+\{(l+1) \Gamma+2 U\} \bar{j}\right] . }
\end{aligned}
$$

The relation between $k_{A}$ and $\Pi$ is given for $l \geq 1$ by

$$
\begin{aligned}
& (l-1)(l+2) k_{u}=16 \pi R^{2} L_{u}+\left(R^{4} \Pi\right)_{, u}-e^{\psi-\lambda / 2}\left(R^{4} \Pi\right)_{, x}, \\
& (l-1)(l+2) k_{x}=16 \pi R^{2} L_{x}-\left(R^{4} \Pi\right)_{, x} .
\end{aligned}
$$

For $l \geq 2$, the gauge-invariant metric perturbations are obtained from Eqs. (3.18), (3.19) and (3.20). However, for $l=1$, Eqs. (3.19) and (3.20) give

$$
R^{4} \Pi=16 \pi J
$$

where we assume that the perturbation is regular at $R=0$. Equation (3.21) implies that there is no gravitational-wave mode for $l=1$. 


\section{Field equations: exterior}

We have two metric perturbations $k_{T}$ and $k_{R}$ in the exterior. Defining the master variable $\Pi$ as

$$
\Pi \equiv\left(\frac{k_{R}}{R^{2}}\right)_{, T}-\left(\frac{k_{T}}{R^{2}}\right)_{, R},
$$

we find the following wave equations for $l \geq 2$

$$
-\Phi_{, T T}+\Phi_{, R_{*} R_{*}}-V(R) \Phi=0,
$$

where

$$
\begin{aligned}
\Phi & \equiv R^{3} \Pi \\
V(R) & \equiv\left(1-\frac{2 M}{R}\right)\left(\frac{l(l+1)}{R^{2}}-\frac{6 M}{R^{3}}\right) .
\end{aligned}
$$

Here, we note that $\Phi$ is related to the variable $\tilde{\psi}$ defined in [22] as $\tilde{\psi}=l(l+1) \Phi$. Using the double-null coordinates $(\bar{u}, \bar{v})$, we obtain

$$
4 \Phi_{, \bar{u} \bar{v}}+V(R) \Phi=0
$$

Equation (3.23) has a static solution with an appropriate fall-off at infinity as

$$
\Phi_{\text {static }}=\frac{q}{l(l+1)}\left(\frac{2 M}{R}\right)^{l} F\left(l-1, l+3,2 l+2 ; \frac{2 M}{R}\right),
$$

where $F(a, b, c ; z)$ denotes the hypergeometric function and $q$, which has the dimension of length, corresponds to the multipole moment of the system. Because $[l(l+1)]^{-1}$ is factorized out in the above equation, the definition of the moment is the same as that defined in [22]. This static solution is used for providing initial conditions of metric perturbations (see Sec. V).

The relation between $k_{A}$ and $\Pi$ is given for $l \geq 1$ by

$$
\begin{aligned}
(l-1)(l+2) k_{T} & =-\left(1-\frac{2 M}{R}\right)\left(R^{4} \Pi\right)_{, R} \\
& =\left(R^{4} \Pi\right)_{, \bar{u}}-\left(R^{4} \Pi\right)_{, \bar{v}} \\
(l-1)(l+2) k_{R} & =-\left(1-\frac{2 M}{R}\right)^{-1}\left(R^{4} \Pi\right)_{, T} \\
& =-\left(1-\frac{2 M}{R}\right)^{-1}\left[\left(R^{4} \Pi\right)_{, \bar{u}}+\left(R^{4} \Pi\right)_{, \bar{v}}\right] .
\end{aligned}
$$

For $l=1$, we find the solutions of Eqs. (3.28) and (3.29) as

$$
R^{4} \Pi=\text { const. }
$$

The integration constant in Eq. (3.30) is related to the total angular momentum of the fluid perturbation for $l=1$. The master variable $\Pi$ is time independent in the exterior for $l=1$ as shown in Eq. (3.30). This constancy implies the conservation of the angular momentum of linear perturbation in the spherically symmetric background.

To compute nonspherical metric perturbations, we divide the background spacetime into regions I-III (see Fig. 11). Region I is defined as the interior of the star. Region II is an intermediate exterior region from which one can emit an ingoing null ray that encounters the stellar surface before the stellar surface is swallowed into the event horizon. Region III is the exterior region outside region II. Region II is introduced to help the matching procedure at the stellar surface in numerical computation. Hereafter, we will refer to the ingoing null surface which divides the exterior regions into regions II and III as the junction null surface. Such an elaborate procedure is needed to calculate the late-time gravitational radiation extracted at the point far from the star and to assure that the matching condition is satisfied at the stellar surface simultaneously.

For the convenience of computation, we introduce new null coordinates $\tilde{u}$ and $\tilde{v}$ in region II. We identify these null coordinates $\tilde{u}$ and $\tilde{v}$ with the values of the proper time $\tau_{\mathrm{s}}$ of an observer comoving at the stellar surface $x=x_{\mathrm{s}}$ at its 
intersection of an outgoing ray $(\tilde{u}=$ const) and an ingoing ray $(\tilde{v}=$ const $)$, respectively. Namely, the stellar surface is given by $\tilde{u}=\tilde{v}$.

When we define functions $A$ and $B$ as

$$
\begin{aligned}
& A(\tilde{u}) \equiv \frac{d \bar{u}}{d \tilde{u}}=\frac{1}{\Gamma+U}\left(\tilde{u}, x_{\mathrm{s}}\right), \\
& B(\tilde{v}) \equiv \frac{d \bar{v}}{d \tilde{v}}=\frac{1}{\Gamma-U}\left(\tilde{v}, x_{\mathrm{s}}\right),
\end{aligned}
$$

we can rewrite the wave equation for $\Phi$ as

$$
4 \Phi_{, \tilde{u} \tilde{v}}+A(\tilde{u}) B(\tilde{v}) V(R) \Phi=0 .
$$

We integrate Eq. (3.33) in region II. The event horizon is given by a finite value of $\tilde{u}$. It is found that the effective potential term $\tilde{V} \equiv A(\tilde{u}) B(\tilde{v}) V(R)$ is regular on the event horizon in this coordinate system, which helps numerical integration of the wave equation.

\section{Matching}

The matching condition at the stellar surface $x=x_{\mathrm{s}}$ for the odd-parity perturbations is obtained from the continuity condition for $\Pi, n^{A} L_{A}$, and $n^{A} \Pi_{\mid A}-16 \pi R^{-2} u^{A} L_{A}$ for $l \geq 1$, and for $n^{A} k_{A}$ and $u^{A} k_{A}$ for $l \geq 2$, where $n_{A} \equiv$ $-\epsilon_{A B} u^{B}[24]$. The explicit equations are

$$
\begin{aligned}
& \Pi_{\mathrm{in}}=\Pi_{\mathrm{ex}}, \\
& -e^{-\psi} \Pi_{\mathrm{in}, u}+e^{-\lambda / 2} \Pi_{\mathrm{in}, x}-16 \pi R^{-4} e^{-\lambda / 2} j \\
= & -\frac{\Pi_{\mathrm{ex}, \bar{u}}}{\Gamma+U}+\frac{\Pi_{\mathrm{ex}, \bar{v}}}{\Gamma-U}=-\Pi_{\mathrm{ex}, \tilde{u}}+\Pi_{\mathrm{ex}, \tilde{v}} .
\end{aligned}
$$

Using $u^{A} \Pi_{\mathrm{in} \mid A}=u^{A} \Pi_{\mathrm{out} \mid A}$, we can derive an alternative form of the matching condition as

$$
\begin{aligned}
& -2 e^{-\psi} \Pi_{\mathrm{in}, u}+e^{-\lambda / 2} \Pi_{\mathrm{in}, x}-16 \pi R^{-4} e^{-\lambda / 2} j \\
= & -2 \frac{\Pi_{\mathrm{ex}, \bar{u}}}{\Gamma+U}=-2 \Pi_{\mathrm{ex}, \tilde{u}} .
\end{aligned}
$$

For $l=1$, the matching conditions lead to

$$
\begin{aligned}
& \Pi_{\mathrm{in}}=16 \pi \frac{J(x)}{R^{4}}, \\
& \Pi_{\mathrm{ex}}=16 \pi \frac{J\left(x_{\mathrm{s}}\right)}{R^{4}} .
\end{aligned}
$$

Thus, the gauge-invariant variable $\Pi$ is completely determined by the initial distribution of perturbed angular momentum in the star.

\section{GRAVITATIONAL WAVES}

To compute gravitational waves in the wave zone, it is convenient to adopt the radiation gauge. In this gauge, the following tetrad components denote the + and $\times$ modes of gravitational waves;

$$
\begin{aligned}
h_{+} & \equiv \frac{1}{2}\left(h_{\hat{\theta} \hat{\theta}}-h_{\hat{\phi} \hat{\phi}}\right), \\
h_{\times} & \equiv h_{\hat{\theta} \hat{\phi}} .
\end{aligned}
$$

Hereafter, we adopt the following choice for the orthonormal bases:

$$
\begin{aligned}
& Y_{l 0} \text { for } m=0 \text { and } \\
& Y_{l, m_{ \pm}} \equiv \frac{1}{\sqrt{2}}\left(Y_{l, m} \pm Y_{l,-m}\right) \text { for } m \neq 0
\end{aligned}
$$


The metric perturbations $h_{+}$and $h_{\times}$in the radiation gauge are written in terms of $\Phi$ as

$$
\begin{aligned}
& h_{+}=-\frac{1}{(l-1)(l+2)} \frac{1}{R}(\Phi+\text { const }) \mathcal{A}_{+}(\theta, \phi)+O\left(R^{-2}\right), \\
& h_{\times}=-\frac{1}{(l-1)(l+2)} \frac{1}{R}(\Phi+\text { const }) \mathcal{A}_{\times}(\theta, \phi)+O\left(R^{-2}\right),
\end{aligned}
$$

where the angular dependence can be explicitly calculated as

$$
\begin{aligned}
\mathcal{A}_{+}(\theta, \phi) & \equiv S_{\theta: \theta}-\frac{1}{\sin ^{2} \theta} S_{\phi: \phi} \\
& =2\left(\frac{1}{\sin \theta} Y_{, \theta \phi}-\frac{1}{\sin \theta \tan \theta} Y_{, \phi}\right), \\
\mathcal{A}_{\times}(\theta, \phi) & \equiv \frac{S_{\theta: \phi}+S_{\phi: \theta}}{\sin \theta} \\
& =\frac{2}{\tan \theta} Y_{, \theta}-2 m^{2} \frac{1}{\sin ^{2} \theta} Y+l(l+1) Y .
\end{aligned}
$$

Here, $Y$ denotes one of the bases shown in Eq. (4.3). The luminosity of gravitational waves $P_{l, n}$ for each mode in terms of the master variable is given by (see [22, 33] for $m=0$ and also [44])

$$
\begin{aligned}
\frac{d P_{l, n}}{d \Omega} & =\frac{1}{16 \pi(l-1)^{2}(l+2)^{2}} \Phi_{, \bar{u}}^{2}\left(\mathcal{A}_{+}^{2}+\mathcal{A}_{\times}^{2}\right)(\theta, \phi), \\
P_{l, n} & =\frac{1}{16 \pi} \frac{l(l+1)}{(l-1)(l+2)} \Phi_{, \bar{u}}^{2},
\end{aligned}
$$

where the subscript $n$ denotes 0 and $m_{ \pm}$.

\section{NUMERICAL METHOD}

\section{A. Numerical integration}

The spherically symmetric stellar collapse is computed using the single-null comoving coordinates. Our method is essentially the same as that used by Baumgarte, Shapiro, and Teukolsky [39]. An artificial viscosity term is incorporated to deal with shock waves. The details of numerical method which we adopt are found in [39].

To solve the perturbation equations for the interior of the star (region I), it is convenient to decompose Eq. (3.18) into the first-order differential equations as

$$
\begin{aligned}
& -2 e^{-\psi-\lambda / 2} Q_{, u}+e^{-\psi-\lambda / 2}\left(e^{\psi-\lambda / 2} Q\right)_{, x}+\frac{2(l+1)}{R}\left\{-(\Gamma+U) e^{-\psi} P+\Gamma e^{-\lambda / 2} Q\right\} \\
& -(l+2)\left[4 \pi(\epsilon-p)+(l-2) \frac{2 m}{R^{3}}\right] \bar{\Pi} \\
& =16 \pi\left[R e^{-\psi-\lambda / 2}\left(e^{\psi} \bar{j}\right)_{, x}+\{(l+1) \Gamma+2 U\} \bar{j}\right], \\
& -2 e^{-\psi-\lambda / 2} P_{, x}+e^{-\psi-\lambda / 2}\left(e^{\psi-\lambda / 2} Q\right)_{, x}+\frac{2(l+1)}{R}\left\{-(\Gamma+U) e^{-\psi} P+\Gamma e^{-\lambda / 2} Q\right\} \\
& \quad-(l+2)\left[4 \pi(\epsilon-p)+(l-2) \frac{2 m}{R^{3}}\right] \bar{\Pi} \\
& =16 \pi\left[R e^{-\psi-\lambda / 2}\left(e^{\psi} \bar{j}\right)_{, x}+\{(l+1) \Gamma+2 U\} \bar{j}\right], \\
& \bar{\Pi}_{, u}=P \\
& \bar{\Pi}_{, x}=Q .
\end{aligned}
$$

We note that the regularity at the center requires

$$
P=e^{\psi-\lambda / 2} Q .
$$


Equation (5.1) constitutes a hyperbolic-type partially differential equation to which we apply the first-order up-wind scheme to stably evolve the function $Q$. Other equations constitute ordinarily differential equations, and thus the integration is carried out with the second-order Runge-Kutta method. The Courant-Friedrich-Lewy (CFL) condition for the stability of integration limits the $n$-th time step $\Delta u^{n}$ as

$$
\Delta u^{n}=2 C \min _{i} e^{-\psi_{i}^{n}+\left(\lambda_{i}^{n} / 2\right)} \Delta x_{i},
$$

where $C(\leq 1)$ is the Courant number.

In the exterior of the star (regions II and III), the double-null coordinates are adopted. In region II, Eq. (3.33) is decomposed into the first-order differential equations as

$$
\begin{aligned}
\tilde{Z}_{, \tilde{v}} & =-\frac{1}{4} A(\tilde{u}) B(\tilde{v}) V(R) \Phi, \\
\tilde{W}_{, \tilde{u}} & =-\frac{1}{4} A(\tilde{u}) B(\tilde{v}) V(R) \Phi \\
\Phi_{, \tilde{u}} & =\tilde{Z} \\
\Phi_{, \tilde{v}} & =\tilde{W}
\end{aligned}
$$

and in region III, Eq. (3.26) is decomposed as

$$
\begin{aligned}
Z_{, \bar{v}} & =-\frac{1}{4} V(R) \Phi, \\
W_{, \bar{u}} & =-\frac{1}{4} V(R) \Phi, \\
\Phi_{, \bar{u}} & =Z, \\
\Phi_{, \bar{v}} & =W .
\end{aligned}
$$

For integration of these equations, we use the finite differencing scheme proposed by Hamadé and Stewart [45].

The coordinates $(\tilde{u}, \tilde{v})$ depend on the spacetime trajectory of the stellar surface. Thus, they are determined after the spherically symmetric stellar dynamics is solved. For this reason, we divide a numerical simulation into two steps. In the first step, we carry out numerical computation for the zeroth-order background solution taking into account the CFL condition for the first-order nonspherical perturbations, and in the second step, we evolve the nonspherical perturbations.

For accurate numerical integration, the distribution of grid points in region I plays a quite important role. We adopt the equally spaced grid in terms of the initial circumferential radius. In region II, the distribution of grid points is automatically determined in computing zeroth-order solution. In region III, the equally spaced grid in terms of $\bar{u}$ and $\bar{v}$ works well.

\section{B. Matching}

At the stellar surface, the matching conditions (3.34) and (3.36) for $\Pi$ and its derivatives determine the boundary conditions for $\bar{\Pi}, P$, and $Q$ at $x=x_{\mathrm{s}}$ in region I, and for $\Phi, \tilde{Z}$ and $\tilde{W}$ at $\tilde{u}=\tilde{v}$ in region II. In matching solutions of regions I and II, we have to be careful since the outermost several mass shells form a very disperse envelope during the collapse. This implies that the grid resolution around the outermost envelope is not very good. If the matching between regions I and II is done at the location of the outermost mass shell, a large numerical error is produced. To suppress the numerical error, we match the solutions at the location of a mass shell which is not outermost one but is located slightly inside the outermost one. We find that this works quite well. We have also checked that the numerical results do not depend on the location of the matching if more than $\sim 95 \%$ of the total mass is contained inside the mass shell for the matching.

At the junction null surface, the matching is not necessary, since the double-null coordinates are adopted in both regions. We only need to store the numerical data set on the junction null surface in region II, and use it as a part of initial data for the integration of region III.

\section{Initial data}

In the present formulation, the null cone composed of $u=u_{0}, \tilde{u}=\tilde{u}_{0}$, and $\bar{u}=\bar{u}_{0}$ should be taken as an initial null surface. Since it is the characteristic surface of the wave equation, we only need to specify $\Pi_{\text {init }}(R)$ and $\beta_{\text {init }}(R)$ there. 
We provide the initial data set in the following manner: In region I, we first give the functions $\Pi_{\text {init }}(R)$ and $\beta_{\text {init }}(R)$. Then, $\bar{\Pi}\left(u_{0}, x\right)$ and $j(x)$ are specified from Eqs. (3.10) and (3.17). From the initial data $\bar{\Pi}\left(u_{0}, x\right)$, we can determine $Q\left(u_{0}, x\right)$ on the initial slice by differentiation through Eq. (5.4). Then, from $\bar{\Pi}\left(u_{0}, x\right), Q\left(u_{0}, x\right)$, and $j(x)$, we can obtain $P\left(u_{0}, x\right)$ by integrating Eq. (5.2) with the central value given by the boundary condition (5.5) at the center.

In region II we first provide $\Phi\left(\tilde{u}_{0}, \tilde{v}\right)=\Pi\left(\tilde{u}_{0}, \tilde{v}\right) R^{3}\left(\tilde{u}_{0}, \tilde{v}\right)$. Then, $\tilde{W}\left(\tilde{u}_{0}, \tilde{v}\right)$ is obtained by differentiation through Eq. (5.10), and $\tilde{Z}$ by integrating Eq. (5.7) with the initial value given by the matching condition (3.36) at the surface.

In region III the method for construction of the initial data sets of $\Phi, W$, and $Z$ is the same as that in region II, except for that the initial values needed for integration of Eq. (5.11) are given at the point on the junction null surface.

When the background spacetime is initially momentarily static, it is natural to choose momentarily static initial data sets. Thus, the initial data set is given in the following manner. First we specify $\beta_{\text {init }}(R)$, and compute $j(x)$ from Eq. (3.10). Then, we determine $\bar{\Pi}\left(u_{0}, x\right)$ and $Q\left(u_{0}, x\right)$ by integrating Eqs. (5.2) and (5.4) using the condition $P\left(u_{0}, x\right)=P_{, x}\left(u_{0}, x\right)=0$ with the initial value $\bar{\Pi}\left(u_{0}, 0\right)$ and $Q\left(u_{0}, 0\right)=0$. Here, we have to tune $\bar{\Pi}\left(u_{0}, 0\right)$ so that it matches the static exterior solution at the stellar surface. The matching is written as

$$
e^{-\lambda / 2} Q+\left[(2 l+1)+\frac{2 M}{R} \frac{F_{l}^{\prime}}{F_{l}}\right] \frac{\Gamma+U}{R} \bar{\Pi}-16 \pi \bar{j} R=0,
$$

where $F(l-1, l+3,2 l+2 ; 2 M / R)$ is abbreviated as $F_{l}$.

After this procedure, the multipole moment $q$ is determined by matching the interior solution with the exterior static solution (3.27). Then, using the moment $q$, the initial data set for the exterior solution is provided by Eq. (3.27).

\section{Event horizon}

The effective potential term of the wave equation for $\Pi$ is regular on the event horizon. However, it contains a term of the form "zero divided by zero" (for $R \rightarrow 2 M, A \rightarrow \infty$, and $V \rightarrow 0$ ). This implies that it is not possible to numerically integrate the wave equation for $\Pi$ on the null surfaces which are very close to the event horizon. To integrate the wave equations until the null surface reaches the event horizon, we use an extrapolation for the value of $\Pi$ on the junction null surface in the neighborhood of the event horizon as

$$
\Pi=\Pi^{N_{\max }}+\frac{\Pi^{\mathrm{EH}}-\Pi^{N_{\max }}}{R^{\mathrm{EH}}-R^{N_{\max }}}\left(R-R^{N_{\max }}\right),
$$

where the value $\Pi^{\mathrm{EH}}$ on the event horizon is estimated as

$$
\Pi^{\mathrm{EH}}=\Pi^{N_{\max }}+\frac{\Pi^{N_{\max }}-\Pi^{N_{\max }-1}}{R^{N_{\max }}-R^{N_{\max }-1}}\left(R^{\mathrm{EH}}-R^{N_{\max }}\right),
$$

$\Pi^{N_{\max }}$ and $R^{N_{\max }}$ are the values for $\Pi$ and $R$ on the junction null surface at the $N_{\max }$ th (final) time step in region II and $R^{\mathrm{EH}}=2 M$. To compute gravitational waves in region III emitted in the neighborhood of the event horizon, this extrapolation is necessary and quite helpful.

\section{CODE TESTS}

\section{A. Spherically symmetric hydrodynamic code}

As the first test of the spherically symmetric general relativistic hydrodynamic code, we performed a simulation for the homogeneous dust collapse. The numerical solution can be compared with the Oppenheimer-Snyder solution [28]. We chose the total grid number as 500 in this test. As the initial condition, we set a momentarily static dust ball of uniform density and of radius $4 M$. Precisely speaking, the dust surface is maximally expanded on the initial null slice. In this case, the event horizon is located at $\tau_{\mathrm{s}}=7.243 M$, where $\tau_{\mathrm{s}}$ denotes the proper time of an observer comoving with the stellar surface.

In Fig. 2 we show the time evolution of the coordinate velocity profiles as a function of circumferential radius at selected time steps. The crosses denote the numerical results and the solid curves the exact solutions. The figure shows that the numerical results agree with the exact solutions within $0.01 \%$ error. We also note that the computation can be continued until the null hypersurface reaches a surface very close to the event horizon. 
To demonstrate that the code works well even in the presence of pressure, we carried out a long-term evolution of spherically symmetric stars in equilibrium. Here, we adopt the polytropic equations of state as

$$
\begin{aligned}
\epsilon & =n(1+e), \\
p & =K n^{\Gamma_{\mathrm{a}}}, \\
n e & =\frac{p}{\Gamma_{\mathrm{a}}-1},
\end{aligned}
$$

where $e$ is the specific internal energy, and $\Gamma_{\mathrm{a}}$ an adiabatic constant, for which we choose as 2 and $\approx 4 / 3$. With $\Gamma_{\mathrm{a}}=2$, moderately stiff equations of state for neutron stars are qualitatively approximated. With $\Gamma_{\mathrm{a}} \approx 4 / 3$, the equation of state for supermassive stars of mass $\gtrsim 10^{6} M_{\odot}$, in which the radiation pressure dominates over the gas pressure, is well approximated. According to [15], the equation of state for supermassive stars may be approximated by the polytropic equation of state with $\Gamma_{\mathrm{a}}$ as

$$
\Gamma_{\mathrm{a}}=\frac{4}{3}+0.00142\left(\frac{M}{10^{6} M_{\odot}}\right)^{-1 / 2} .
$$

Thus, we adopt $\Gamma_{\mathrm{a}}=(4 / 3)+0.00142$ to model a supermassive star of mass $10^{6} M_{\odot}$. The equilibrium configurations are obtained by solving the Tolman-Oppenheimer-Volkoff equation.

In Fig. 3 the gravitational mass as a function of the central density for the equilibrium configurations with $\Gamma_{\mathrm{a}}=$ $(4 / 3)+0.00142$ and 2 are shown. For simulations in this paper, we adopted four static configurations of nearly maximum mass along the equilibrium sequences (see A-D in Fig. 3) as initial data sets. Models A and $\mathrm{C}$ are stable against gravitational collapse, while B and D are marginally stable. We summarize these models in Table $\square$

TABLE I: Models for equilibrium solutions. The unit of $c=G=M=1$ is adopted.

\begin{tabular}{c|ccc}
\hline \hline Model & $\Gamma_{\mathrm{a}}$ & Central energy density & Radius \\
\hline $\mathrm{A}$ & $(4 / 3)+0.00142$ & $1.852 \times 10^{-9}$ & $1.900 \times 10^{3}$ \\
$\mathrm{~B}$ & $(4 / 3)+0.00142$ & $3.707 \times 10^{-9}$ & $1.508 \times 10^{3}$ \\
$\mathrm{C}$ & 2 & $5.944 \times 10^{-3}$ & 5.501 \\
$\mathrm{D}$ & 2 & $1.044 \times 10^{-2}$ & 4.745 \\
\hline \hline
\end{tabular}

For long-term test simulations, we adopt models A and C. To induce a small oscillation, we initially increased the specific internal energy $e$ uniformly by $1 \%$ both for model A and for model C. In Fig. 团 we show the time evolution of the central density. It is found that the stars oscillate in a periodic manner. By performing the Fourier analysis, we measured the period of this stellar oscillation, and found that the oscillation angular frequency is

$$
\omega \approx \bar{\omega} \frac{M^{1 / 2}}{R^{3 / 2}},
$$

where $\bar{\omega}=9.59 \times 10^{-2}$ for model $\mathrm{A}$ and 0.788 for model $\mathrm{C}$, where the initial stellar radii are given by $1.900 \times 10^{3} \mathrm{M}$ and $5.501 M$ for models A and C, respectively (see Table I).

According to a post-Newtonian theory, the angular frequency of the radial oscillation for supermassive stars can be estimated as [15]

$$
\omega^{2}=\frac{3|W|}{I}\left(\Gamma_{\mathrm{a}}-\Gamma_{\text {crit }}\right),
$$

where $W, I$, and $\Gamma_{\text {crit }}$ are the gravitational energy, moment of inertia, and critical polytropic index, respectively. Here, $\Gamma_{\text {crit }}$ is estimated in the post-Newtonian approximation as [46]

$$
\Gamma_{\text {crit }}=\frac{4}{3}+1.125\left(\frac{2 M}{R}\right) .
$$

From this analysis, $\bar{\omega}$ should be $9.62 \times 10^{-2}$ for model A. Thus, the numerical result agrees with the analytic one within $0.5 \%$ error.

The angular frequency of the radial oscillation for neutron stars can be also calculated using the semianalytic formula derived by Chandrasekhar [46]. From this approximate formula, we can predict the oscillation frequency should be 
$\approx 0.9 \rho_{c}^{1 / 2}\left[47\right.$ and hence $\omega \approx 0.063 M^{-1}$. On the other hand, the numerical result indicates that $\omega \approx 0.0616 M^{-1}$, which is again in good agreement with the analytic result within a few percent error.

Models B and D are marginally stable against gravitational collapse. Thus, if the internal energy is reduced, they start collapsing. We have checked that they indeed collapse to black holes. The detailed results of the gravitational collapse are described in Sec. VII

\section{B. Perturbation code}

We have carried out a wide variety of test simulations for our perturbation code. First, we checked that gravitational waves accurately propagate on the flat Minkowski background. For $l=2$, the exact solutions for linear gravitational waves in the Minkowski spacetime are obtained as [48]

$$
\bar{\Pi}=3 \frac{I(t-r)-I(t+r)}{r^{5}}+3 \frac{I^{\prime}(t-r)+I^{\prime}(t+r)}{r^{4}}+\frac{I^{\prime \prime}(t-r)-I^{\prime \prime}(t+r)}{r^{3}},
$$

where $I$ is an arbitrary function, and $I^{\prime}$ its derivative. Here, we choose

$$
I(y)=\exp \left[-4(y-2)^{2}\right]
$$

Since the background spacetime is flat, the matching surface between regions I and II is artificial. As the matching surface, we chose the surface of $r=1$ in this test. In Fig. 5 we show numerical results with the exact solution for the waveforms observed at $r=5$. To demonstrate the convergence of the numerical solutions, we performed simulations with three levels of the grid resolution as $\Delta r=\Delta u=\Delta v=0.001,0.002$, and 0.004. Figure [5 shows that numerical results converge at first order to the exact solution. We note that the number of grid points per wavelength is $\sim 1000$ for the best-resolved case.

Next, we computed the propagation of gravitational waves for $l=2$ in the collapse of a homogeneous dust ball. The same type of computation was already carried out [22, 23], so that we can calibrate our code by comparing the present results with previous ones.

In [22], the momentarily static perturbations are provided at the initial hypersurface. Thus, we also choose the momentarily static initial data on the null hypersurface (i.e., the dust surface is assumed to reach the maximum expansion at initial slice). However, the word of caution is appropriate here. The previous computation was carried out using $1+1$ coordinate system (not single-null coordinate system). On the other hand, we choose the single-null coordinate system. Namely, the coordinate system and also the time slicing are different between the two. Thus, even if we set the same function of $\bar{\beta}$ in different coordinate systems, this does not imply that we give the identical initial condition. Moreover, the former formulation has ambiguity in determining the second-order time derivative of gravitational perturbation, while the latter one does not. Hence, the momentarily static initial data sets for these two formulations are different from each other. It implies that it is difficult to precisely compare the results obtained in these two formulations.

To calibrate the effect of different initial conditions on the results, we provided two perturbation profiles fixing $q$. In the first one, we gave $\bar{\beta}_{\text {init }}(R)=$ const on the initial hypersurface, and in the second one, $\bar{\beta}_{\text {init }}(R) \propto \exp \left[-\left(R / R_{c}\right)^{2}\right]$, where $R_{c}$ is chosen to be one-third of the initial surface radius.

In Fig. [6] the total radiated energy of gravitational waves are summarized. In these numerical computations, gravitational waves are extracted at $R=40 M$. For comparison, we also plot the results of 22]. We note that in this figure, $q$ is normalized so that $q=2 M$. It is found that numerical results of two different profiles of $\beta$ differ by a factor of $\sim 1.4$, and that the results of $[22$ are greater than our results by a factor of $\sim 3$. However, the total radiated energy systematically decreases as the initial dust radius increases for all the cases in the same manner. Thus, we conclude that our numerical results agree with the previous ones besides a possible systematic error associated with the difference of the coordinate conditions.

The waveform, luminosity, and integrated total energy of gravitational waves from a collapsing dust ball with the initial radius $R=20 M$ for $l=2$ are plotted in Fig. 7 Gravitational waves are extracted at $R=40 M$. As found in [22], the quasinormal ringing oscillation and subsequent power-law tail characterize the gravitational waveforms. The complex frequency of the fundamental quasinormal mode was calculated to be $2 M \omega=0.74734+0.17792 i$ by solving the eigenvalue equations 49]. On the other hand, our numerical results show that the complex frequency of the damped oscillation is given by $2 M \omega=0.752+0.179 i$. Thus, the numerical results agree with the theoretical value within $1 \%$ error both for the frequency and for the damping rate. It is found that the waveform is characterized by the power-law tail for $\bar{u} \gtrsim 350 M$. The power-law index numerically computed is $\approx 7.0$ and also agrees with that analytically derived in [50] as $-(2 l+3)=-7$.

We also computed gravitational waves from a static star. As the stellar models, we adopted models A and C. Since the odd-parity matter perturbation is time independent in this case, gravitational waves propagate freely. This implies 
that with the momentarily static initial perturbation, no gravitational radiation should be emitted. We checked that this is indeed the case except for the tiny amount emitted soon after the simulation started, which is due to numerical errors associated with the finite differencing and relaxation of the numerical system.

\section{STELLAR COLLAPSE TO BLACK HOLES AND GRAVITATIONAL WAVES}

We have computed stellar collapse of models B and D to black holes. Since they are marginally stable against gravitational collapse, we extracted the internal energy $e$ by $1 \%$ initially to induce the collapse. Here the energy extraction is done on the initial null cone. The difference between the energy extractions on the initial null cone and on the spacelike hypersurface is very small for nonrelativistic stars but may be significant for highly relativistic stars. During the collapse, we adopt the $\Gamma$-law equation of state as

$$
p=\left(\Gamma_{\mathrm{a}}-1\right) n e
$$

The simulations were carried out using both the May-White and the Hernandez-Misner schemes. We note that with the latter scheme, we can follow the evolution only outside an event horizon. This implies that we cannot find apparent horizon and event horizon in the following simulation. We stop the calculation of the stellar collapse and estimate the value of the perturbation field on the event horizon using the extrapolation when $R_{\mathrm{s}} / 2 M=1.01$ is satisfied for the surface radius $R_{\mathrm{s}}$. We have confirmed that the obtained waveform is not so sensitive to the choice of the criterion. In the simulation of the collapse of models B and D, we have observed no evident shock wave until the black hole forms.

\section{A. Collapse of a supermassive star}

Supermassive stars are quasistable objects of mass $5 \times 10^{5} M_{\odot} \lesssim M \lesssim 10^{10} M_{\odot}$ and possible direct progenitors of supermassive black holes [14, 15]. They quasistationarily contract due to radiative cooling to the onset of radial instability [14, 15], resulting in formation of supermassive black holes. The quasistatic evolution of rotating supermassive stars was recently investigated by Baumgarte and Shapiro [16]. Taking into account that supermassive stars are likely to be rigidly rotating and that the adiabatic index is $\approx 4 / 3$, they clarified that the ratio of the rotational energy to the gravitational energy is at most $\sim 0.009$ at the onset of the gravitational collapse. They also found that oblateness of supermassive stars around the central region is very small (see Fig. 1 in [16]), justifying that the Roche model 15] for rotating supermassive stars is adequate. Shibata and Shapiro 19 numerically computed the collapse of a marginally stable rotating supermassive star to a Kerr black hole in the two-dimensional fully general relativistic simulation. They indicated that more than $90 \%$ of the stellar mass collapses directly into the black hole in the dynamical time scale as in the spherical collapse. These results suggest that if we pay attention only to the inner region, the collapse proceeds in a nearly spherical manner. Motivated by this fact, we apply the present perturbation analysis to compute gravitational waves emitted during the collapse of supermassive stars.

\section{Spherical collapse}

We adopted model B with $\Gamma_{\mathrm{a}}=(4 / 3)+0.00142$ to model a supermassive star of mass $10^{6} M_{\odot}$. In the numerical simulation, we typically take 1000 grid points to cover the supermassive star. For the collapse of model B, with the May-White scheme, apparent horizon was located near the center in a late time of collapse, but soon after the formation, numerical accuracy deteriorates and as a result computation crashed before the event horizon swallowed all the fluid elements. On the other hand, the computation can be continued until the null hypersurface reaches the event horizon with the Hernandez-Misner scheme; i.e., the whole region outside the event horizon is computed numerically. This clearly indicates the robustness of the Hernandez-Misner scheme. The matching is done on the mass shell within which $99.7 \%$ of the total mass is enclosed. In the following, we deal with this matching surface as the stellar surface.

In Fig. 8 (a) we display the snapshots of the density profile at selected time steps. In this simulation, the spacetime settles down to a static one at $\bar{u} \simeq 176760 M$. The calculation has been stopped at $\bar{u} \simeq 176763 M$ or $\tau_{\mathrm{s}} \simeq 175294 M$. At this moment, we have obtained the redshift $\left(1+z_{\mathrm{s}}\right) \simeq 190.7$ at the surface. Since the equation of state of supermassive stars is soft, the mass is highly concentrated around the center. In the late stage of the collapse, the increase of the central density is accelerated, and hence the collapse proceeds in a runaway manner. (This makes the simulation without null formulation technically difficult.) Figure 8 (b) shows the trajectories of mass shells for $\bar{u} \gtrsim 176400 M$. Each mass shell asymptotically approaches a constant value greater than $2 m$ because the lapse function decreases to zero. This figure shows that the central region collapses earlier, while the outer envelope accretes slowly after 
the evolution of the central region is almost frozen. Figure 8(c) shows the total mass contained in the high-redshift region in which the lapse function is less than 0.1 [or equivalently $(1+z)$ is greater than 10 where $z$ denotes the gravitational redshift]. This region first appears at the center at $\bar{u} \simeq 176510 M$. We find that $80 \%$ of the stellar mass is swallowed into this high-redshift region within the time interval $\Delta \bar{u} \sim 15 M$. After the inner region collapses, surrounding atmosphere falls into this high-redshift region spending a much longer time $\simeq 200 M$.

\section{Gravitational radiation}

For computation of the nonspherical perturbations, a static initial condition for $\bar{\beta}$ should be given. However, the realistic perturbation profile of the initial data set is not clear for the odd-parity perturbation. The purpose of this paper is to study the gravitational waveforms during the formation of a black hole qualitatively. Thus, to investigate the dependence of the gravitational waveforms on the initial perturbation profile, we gave three kinds of the initial data sets as (1) with $\bar{\beta}_{\text {init }}=$ const, $(2)$ with $\bar{\beta}_{\text {init }}=\exp \left[-\left(R / R_{\mathrm{c}}\right)^{2}\right]$, and $(3)$ with $\bar{\beta}_{\text {init }}=\exp \left\{-\left[\left(R-R_{\mathrm{s}}\right) / R_{\mathrm{c}}\right]^{2}\right\}$, where the scale length of the inhomogeneity, $R_{\mathrm{c}}$, for the matter perturbation is chosen to be $R_{\mathrm{c}}=R_{\mathrm{s}} / 3$. For $(1)$, the perturbation is uniformly distributed. For (2), the amplitude of the perturbation in the inner region is larger than that in the outer region. For (3), the amplitude of the perturbation is the largest near the stellar surface.

To check the convergence, computations for case (1) were carried out with $N=1000,500$ and 250 , where $N$ is the number of grid points in the stellar interior. The wavelength of gravitational waves is roughly comparable with the stellar size for the early stage and with the stellar size or the size of the formed black hole for the late stage. Since we adopt the comoving coordinate, the number of grid points per wavelength is kept almost constant and of the order 1000 for 1000-zone calculations.

The waveform, luminosity, and integrated total energy of gravitational waves for $l=2$ are plotted in Fig. 9. Since the oscillation period of gravitational waves is much shorter than the total time of integration, we show them only around the time of major emission. Figure 9 demonstrates that the convergence is achieved well. For example, it is found that the totally radiated energy shows the first-order convergence. Although the numerical error in the time of the major emission might look very large, it is only due to the extremely long time for the collapse compared to the duration of the major emission. It is seen in Fig. 9/(a) that the waveform is made up of the precursory wave, quasinormal ringing, long-time-scale decay, quasinormal ringing again, and power-law tail. Figures 9 (b) and (c) indicate that the primary and secondary contributions to the totally radiated energy come from the first and second bursts of quasinormal ringing, respectively. The reason of the existence of this second burst will be explained later.

We show the waveform, luminosity, and integrated total energy of quadrupole $(l=2)$ gravitational waves for different initial perturbations (1)-(3) in Fig. 10. In all three cases, the amplitude of the perturbation is normalized so that $q=2 M$. Here, we display the results with $N=1000$.

As seen in Fig. 10, the gravitational waveform depends strongly on the perturbation profile initially given. For case (2), as seen in Figs. 10 (c) and (d), gravitational waves of high amplitude are emitted around $\bar{u} \simeq 176510 M$, approximately at the same time as the formation of the high-redshift region. The waveform is characterized mainly by a quasinormal mode of the formed black hole. The duration of this major emission is roughly $\sim 50 M$, i.e., approximately equal to the period and/or the damping time of the quasinormal mode. Indeed, the waveform for $\bar{u} \gtrsim 176520 M$ is well fitted by a damped oscillation of the complex frequency $2 M \omega \approx 0.74+0.19 i$, which agrees with the theoretically predicted value $2 M \omega=0.74734+0.17792 i$ 49] within a few percent error. It is possible in principle in the present scheme to observe the modulation of gravitational waveforms due to mass accretion as Papadopoulos and Font [51] demonstrated for the spherically symmetric Klein-Gordon field on the accreting black hole background. However, in the present numerical results, it is difficult to distinguish the effects of the mass increase from the gravitational waveforms, possibly because the mass accretion rate is rather high and the duration of the accretion is not so long.

For case (1), as seen in Figs. [10(a) and (b), gravitational waves look as the linear combination of a quasinormal mode of a black hole and a long-time-scale and nonoscillative component (note that the amplitude of gravitational waves does not settle down to zero for a long-time duration $\simeq 200 M$ after $\bar{u} \simeq 176510 M$ ). The long-time-scale component is produced due both to the perturbation profile initially given and to the nature of the collapse of the background spherical star: In the collapse of supermassive stars, the central region collapses first and subsequently, the outer envelope gradually falls into the black hole spending a long time duration $\simeq 200 M$. Since $\bar{\beta}_{\text {init }}=$ const, the outer envelope retains a considerable fraction of the perturbation for case (1). As a result, the quasinormal modes are likely to be continuously excited for the duration $\simeq 200 M$ during which the matter falls into a black hole. However, the quasinormal modes continuously excited should cancel each other due to the phase cancellation effect [52]. This suppresses the amplitude of gravitational waves and produces a long-time-scale component, which results in the suppression of radiated energy as seen in Fig. 10(g).

For case (3), in which the matter perturbation is retained mainly near the stellar surface, as seen in Figs. 10 (e) 
and (f), the effect of the phase cancellation is more outstanding. In this case, the amplitude of gravitational waves is highly suppressed and the amplitude of the quasinormal mode ringing is much smaller than that of the long-time-scale component. We note that the time scale of the long-time-scale component is in approximate agreement with the time duration in which the accretion of the surrounding envelope continues.

Figure 10(h) shows the one-sided power spectral density for the obtained gravitational waveforms for all three cases. It is clear that the phase cancellation effects significantly suppress the high-frequency component for cases (1) and (3) while the low-frequency component depends not on the initial distribution of matter perturbation but on the initial moment of perturbation.

To explain more clearly why the long-time-scale and nonoscillative component appears, we artificially superimpose the waveform $\Phi(t)$ obtained for case (2) displayed in Fig. 10(c), which is characterized by quasinormal ringing, with the weight factor $w(t)$ as

$$
\int_{-\infty}^{\infty} d t^{\prime} w\left(t^{\prime}\right) \Phi\left(t-t^{\prime}\right)
$$

where $w(t)$ is chosen by trial as

$$
w(t)=\left\{\begin{array}{cc}
8\left(\frac{t}{t_{\mathrm{c}}}\right) \exp \left(-\frac{t}{t_{\mathrm{c}}}\right)+\left(\frac{t}{t_{\mathrm{a}} / 4}\right)\left[\left(\frac{t}{t_{\mathrm{a}} / 4}\right)^{3}+1\right]^{-1} & \text { for } 0 \leq t \leq t_{\mathrm{a}}, \\
0 & \text { for } t<0, t_{\mathrm{a}}<t,
\end{array}\right.
$$

and we set $t_{\mathrm{c}}=2.95 \mathrm{M}$ and $t_{\mathrm{a}}=236 \mathrm{M}$. The $t_{\mathrm{c}}$ and $t_{\mathrm{a}}$ correspond to the time scales of the collapse of the inner region and of the accretion of the outer envelope, respectively. See Fig. 11(c) for the shape of this function. In the above functional form, the first and second terms imitate the effects of inner collapse and subsequent accretion of the outer envelope, respectively. In Fig. 111(a), we display the result for the superimposed waveform. This result is qualitatively the same as that obtained for case (1) displayed in Fig. 10/a). We note that the long-time-scale and nonoscillative component is produced by the long-term superposition of quasinormal ringing, including a precursory "burst" wave.

Next we choose another weight factor $w(t)$ defined as

$$
w(t)=\left\{\begin{array}{cr}
\left(\frac{t}{t_{\mathrm{a}} / 4}\right)\left[\left(\frac{t}{t_{\mathrm{a}} / 4}\right)^{3}+1\right]^{-1} & \text { for } 0 \leq t \leq t_{\mathrm{a}}, \\
0 & \text { for } t<0, t_{\mathrm{a}}<t
\end{array}\right.
$$

and we again set $t_{\mathrm{a}}=236 \mathrm{M}$. See Fig. [11(c) for the shape of this function. This functional form imitates the effect of accretion of the outer envelope alone. In Fig. 111 b), we display the result for the superimposed waveform. This result is qualitatively the same as that obtained for case (3) displayed in Fig. 10(e). It should be again emphasized that the superposition not of the pure damped oscillation but of the full waveform for case (2), including the precursory burst wave, produces the long-time-scale component. The above consideration confirms our speculation.

We note that the second quasinormal ringing seen around $\bar{u} \simeq 176750 M$ in Figs. 10(a) and (b) for case (1) and in Figs. 10(e) and (f) for case (3) are excited by the stellar surface which falls into the black hole finally. Since the phase cancellation is not effective at this stage, the quasi-normal ringing dominates the waveform for the latest phase. After this ringing, a power-law tail is seen, and the power-law index agrees with the theoretically predicted value -7 .

Finally we note the following point. In this simulation, all the matter is swallowed into a black hole eventually because the zeroth-order solution is spherically symmetric. That is the reason why the accretion ends at some finite moment. However, in a realistic nonspherical problem, the matter around the stellar surface does not fall into the black hole, and would form surrounding disks. This implies that the gravitational waveforms calculated for the latest phase $\bar{u} \gtrsim 176750 M$ will be modified in a realistic simulation.

\section{B. Collapse of a neutron star}

We performed a simulation for the collapse of a neutron star to a black hole adopting model D. As in the collapse of a supermassive star, 1000 grid points are typically taken to cover the neutron star interior. For the collapse of model D, both the May-White and Hernandez-Misner schemes can evolve the collapse to the formation of the event horizon with reasonable accuracy. In the following, the results in the Hernandez-Misner scheme will be described. In this simulation, the matching is done on the mass shell within which $96.1 \%$ of the total mass is enclosed. In the following, we deal with this matching surface as the stellar surface.

In Fig. 12 we show the snapshots of the density profile at selected time steps, trajectory of the mass shells as a function of time, and the mass fraction of the fluid elements in the region of strong gravitational field in which the 
magnitude of the lapse function is less than 0.1. We note that in this simulation, the event horizon is just about to form at $\bar{u} \simeq 111.5 \mathrm{M}$. The calculation has been stopped at $\bar{u} \simeq 111.5 \mathrm{M}$ or $\tau_{\mathrm{s}} \simeq 68.04 \mathrm{M}$. At this moment, we have obtained the redshift $\left(1+z_{\mathrm{s}}\right) \simeq 22.89$ at the surface. In contrast with the collapse of the supermassive stars, the central density increases only by a factor of $\approx 3.0$ throughout the collapse. Thus, the evolution of the central density does not show the runaway behavior. Also, the collapse proceeds very coherently: Figure 12(c) indicates that the high-redshift region first appears at the center at $\bar{u} \simeq 99.1 M$ and that almost all the fluid elements collapse to the high-redshift region in a short time interval $\Delta \bar{u} \sim 6 M$.

To study the evolution of the perturbation, we gave the momentarily static initial data sets with matter perturbations (1)-(3) as in the collapse of a supermassive star. The amplitude of the perturbation is normalized so that $q=2 M$.

In Fig. 13] we show the waveform, luminosity and accumulated energy of gravitational waves for case (1). Computations were carried out with $N=1000,500$, and 250, and Fig. 13] demonstrates that convergence is achieved (note that results with $N=1000$ and 500 agree well with each other). A small modulation is found just after the beginning of computation. This is because the initial data sets which are numerically constructed are not precisely static solutions of the finite differencing equation for the evolution of perturbations. The above is confirmed by the fact that this modulation becomes smaller as $N$ increases. After this spurious precursor disappears, a black hole quasinormal mode is excited and a power-law tail follows [55. No other outstanding feature is found in the gravitational waveform in contrast with the case of the supermassive star collapse. This is likely due to that the collapse proceeds very coherently for $\Gamma_{\mathrm{a}}=2$. The first and major gravitational emission of the quasinormal oscillation occurs approximately just after the appearance of the high-redshift region. From the numerical result, the quasinormal frequency and the index of the power-law tail are calculated as $2 M \omega=0.752+0.176 i$ and -7.1 . These values agree well with the theoretical values $2 M \omega=0.74734+0.17792 i$ and $-(2 l+3)=-7[49$, 50] both within a few percent error.

Figure 14 shows gravitational waves for different initial matter perturbations (1)-(3). The waveform depends only very weakly on the initial perturbation distribution. The reason is that the collapse proceeds in a very coherent manner and hence the difference of the perturbation distribution does not generate outstanding differences. There is no phase cancellation effects on the neutron star collapse irrespective of the shape of the perturbation as opposed to the supermassive star case as is seen in the shape of spectrum. A small time delay of the oscillational phase is seen for cases (1) and (3) compared with case (2). This is simply because the matter perturbation enters the black hole for case (2) earlier than for cases (1) and (3).

\section{SUMMARY}

We have reported a new implementation in linearized Einstein theory. In this code, the Hernandez-Misner scheme is adopted to compute a spherically symmetric zeroth-order solution. As a result, we can compute stellar collapse to a static black hole until the null hypersurface reaches the event horizon, and the whole region outside the event horizon is numerically generated. We emphasize that the collapse of a supermassive star to a black hole proceeds in a runaway manner, i.e., the central density grows rapidly although the surrounding atmosphere does not collapse very rapidly. It is not technically easy to compute such collapse with a $1+1$ numerical scheme. The Hernandez-Misner coordinate system is essential to enable thorough computation of the black hole formation.

We have also proposed a new numerical method to compute gravitational waves in perturbation theory. We divide the computational domain into three regions. For computation of the perturbations in the exterior region, we adopt double-null coordinates which agree with the characteristic curves of gravitational waves. This choice enables the computation of gravitational waves emitted during the entire history of black hole formation.

To study the qualitative nature of gravitational waves from stellar collapse, we performed simulations for the collapse of a supermassive star and a neutron star to black holes. In the gravitational collapse of the neutron star, gravitational waveforms are characterized by a black hole quasinormal mode, as demonstrated in the fully general relativistic simulations $[8]$. On the other hand, for gravitational collapse of the supermassive star, the waveform depends strongly on the perturbation profile that we give initially. For a centrally concentrated matter perturbation, the waveforms are characterized mainly by a black hole quasinormal mode, as in the collapse of neutron stars. However, when the matter perturbation is distributed uniformly, the waveform is determined by a linear combination of the black hole quasinormal mode and a long-time-scale component which results from the superposition of the quasinormal ringing component. Moreover, when the matter perturbation is located around the surface, the long-time-scale component dominates the waveform. This is likely due to the less-coherent nature of the collapse of supermassive stars: the central part collapses earlier and subsequently the outer envelope accretes on to the central black hole.

We have determined the choices of initial distribution of perturbation not assuming physically realistic situations because of the lack of our knowledge on physically realistic odd-parity perturbation. Therefore, at present, we do not claim that the gravitational waves obtained here are realistic in particular for the collapse of supermassive stars. 
However, our present results strongly suggest that the gravitational radiation is not so sensitive to the initial condition for neutron star collapse but is highly sensitive for supermassive star collapse.

In the formation of intermediate-mass black holes (mass $\gtrsim 200 M_{\odot}$ ) formed from quite massive stars, which is destabilized by electron-positron pair creation [53], collapse would proceed in the same manner as for supermassive stars. Thus, gravitational waveforms from the formation of intermediate-mass black holes also depend strongly on the state of the precollapse star.

We have focused on perturbations of odd parity. The dominant modes of gravitational waves are likely to be of even parity in most cases, and thus the study of the even-parity perturbations seems to be more important. In a rotating stellar collapse, quadrupole deformation that rotating stars retain before collapse will be the source of gravitational waves of even parity. The study of such effects on gravitational waves emitted in a black hole formation is now in progress.

\section{Acknowledgments}

We are grateful to K. Nakao and C. Gundlach for helpful discussion. We are also grateful to J.M. Overduin for carefully reading the manuscript. This work was partly supported by the Grant-in-Aid for Scientific Research (Nos. 05540, 11217, 13740143, and 14047207) from the Japanese Ministry of Education, Culture, Sports, Science and Technology.

[1] H. Tagoshi et al., Phys. Rev. D 63, 062001 (2001): M. Ando et al., Phys. Rev. Lett. 86, 3950 (2001).

[2] A. Abramovici et al., Science 256, 325 (1992).

[3] J. Hough, in Proceedings of the Sixth Marcel Grossmann Meeting on General Relativity, edited by H. Sato and T. Nakamura (World Scientific, Singapore, 1992), p. 192.

[4] C. Bradaschia et al, Nucl. Instrum. Methods Phys. Res. A 289, 518 (1990).

[5] K.S. Thorne, in Proceedings of Snowmass 95 Summer Study on Particle and Nuclear Astrophysics and Cosmology, edited by E.W. Kolb and R. Peccei (World Scientific, Singapore, 1995), p. 398.

[6] B.F. Schutz, gr-qc/0111095

[7] H. Dimmelmeier, J. A. Font, and E. Müller, Astron. Astrophys. 393, 523 (2002), and see references cited therein.

[8] R. F. Stark and T. Piran, Phys. Rev. Lett. 55, 891 (1985); in Dynamical Spacetimes and Numerical Relativity, edited by J. M. Centrella (Cambridge University Press, Cambridge, England, 1986), p. 40.

[9] K. Kokkotas and B. Schmidt, Living Rev. Relativ. 2, 4 (1992).

[10] E. W. Leaver, Proc. R. Soc. London A402, 285 (1985).

[11] M.J. Rees, Annu. Rev. Astron. Astrophys. 22, 471 (1984).

[12] M.J. Rees, in Black Holes and Relativistic Stars, edited by R.M. Wald (Chicago University Press, Chicago, 1998), p.79.

[13] M.J. Rees, in Black Holes in Binaries and Galactic Nuclei, edited by L. Kaper, E.P.J. van den Geurel, and P.A. Woudt (Springer-Verlag, New York, 2001), p. 351.

[14] Ya. B. Zel'dovich and I. D. Novikov, Relativistic Astrophysics (University of Chicago Press, Chicago, 1971), Vol. 1.

[15] S. L. Shapiro and S.A. Teukolsky, Black Holes, Neutron Stars and White Dwarfs (Wiley Interscience, New York, 1983).

[16] T. W. Baumgarte and S.L. Shapiro, Astrophys. J. 526, 941 (1999).

[17] M. Shibata, S. L. Shapiro, and K. Uryu, Phys. Rev. D 64, 024004 (2001).

[18] M. Saijo, T.W. Baumgarte, S.L. Shapiro, and M. Shibata, Astrophys. J. 569, 349 (2002).

[19] M. Shibata and S.L. Shapiro, Astrophys. J. Lett. 572, L39 (2002).

[20] S.L. Shapiro and S.A. Teukolsky, Astrophys. J. Lett. 234, L177 (1979).

[21] E.g., M. Shibata and K. Uryu, Phys. Rev. D 61, 064001 (2000): Prog. Theor. Phys. 107, 265 (2002); J. A. Font et al., Phys. Rev. D 64, 084024 (2002).

[22] C.T. Cunningham, R.H. Price, and V. Moncrief, Astrophys. J. 224, 643 (1978); 230, 870 (1979); 236, 674 (1980).

[23] E. Seidel and T. Moore, Phys. Rev. D 35, 2287 (1987); E. Seidel, E.S. Myra, and T. Moore, Phys. Rev. D 38, 2349 (1988); E. Seidel, ibid. 42, 1884 (1990).

[24] C. Gundlach and J.M. Martín-García, Phys. Rev. D 61, 084024 (2000); J.M. Martin-Garcia and C. Gundlach, ibid. 64, $024012(2000)$.

[25] T. Regge and J.A. Wheeler, Phys. Rev. 108, 1063 (1957).

[26] F. J. Zerilli, Phys. Rev. D 2, 2141 (1970).

[27] V. Moncrief, Ann. Phys. (N.Y.) 88, 323 (1974).

[28] J.R. Oppenheimer and H. Snyder, Phys. Rev. 56455 (1939).

[29] U.H Gerlach and U.K Sengupta, Phys. Rev. D 19, 2268 (1979); 22, 1300 (1980).

[30] M.M. May and R.H. White, Phys. Rev. 141, 1232 (1966).

[31] K.A. Van Riper, Astrophys. J. 232, 558 (1979). 
[32] T. Harada, T. Chiba, K. Nakao, and T. Nakamura, Phys. Rev. D 55, 2024 (1997).

[33] H. Iguchi, K. Nakao, and T. Harada, Phys. Rev. D 57, 7262 (1998); H. Iguchi, T. Harada, and K. Nakao, Prog. Theor. Phys. 101, 1235 (1999); 103, 53 (2000).

[34] T. Harada, H. Iguchi, and K. Nakao, Prog. Theor. Phys. 107, 449 (2002).

[35] G. Lemaître, Ann. Soc. Sci. Bruxelles A 53, 51 (1933); R. C. Tolman, Proc. Natl. Acad. Sci. U.S.A. 20, 169 (1934); H. Bondi, Mon. Not. R. Astron. Soc. 107, 410 (1947).

[36] C.W. Misner and D.H. Sharp, Phys. Rev. 136, 571 (1964).

[37] W.C. Hernandez and C.W. Misner, Astrophys. J. 143, 452 (1966).

[38] J.C. Miller and S. Motta, Class. Quantum Grav. 6, 185 (1989).

[39] T.W. Baumgarte, S.L. Shapiro, and S.A. Teukolsky, Astrophys. J. 443, 717 (1995); 458, 680 (1996).

[40] T. Harada, Phys. Rev. D 58, 104015 (1998).

[41] J.C. Niemeyer and K. Jedamzik, Phys. Rev. D 59, 124013 (1999); K. Jedamzik and J.C. Niemeyer, ibid. 59, 124014 (1999).

[42] F. Linke, J.A. Font, H.T. Janka, E. Müller, and P. Papadopoulos, Astron. Astrophys. 376, 568 (2001).

[43] F. Siebel et al., Phys. Rev. D 65, 024021 (2002).

[44] L.D. Landau and E.M. Lifshitz, The Classical Theory of Fields (Pergamon, London, 1975).

[45] R.S. Hamadé and J.M. Stewart, Class. Quantum Grav. 13, 497 (1996).

[46] S. Chandrasekhar, Astrophys. J. 140, 417 (1964).

[47] See, the following references for concrete application of the formula in 46]: M. Shibata, Phys. Rev. D 60, 104052 (1999).

[48] S. A. Teukolsky, Phys. Rev. D 26, 745 (1982).

[49] S. Chandrasekhar and S. Detweiler, Proc. R. Soc. London A 344, 441 (1975).

[50] R.H. Price, Phys. Rev. D 5, 2419 (1972).

[51] P. Papadopoulos and J.A. Font, Phys. Rev. D 63, 044016 (2001).

[52] T. Nakamura and M. Sasaki, Phys. Lett. 106B, 69 (1981).

[53] C. L. Fryer, S. E. Woosley, and A. Heger, Astrophys. J. 550, 372 (2001).

[54] For example, for the marginally bound collapse of an inhomogeneous dust ball, which is described by the Lemaitre-TolmanBondi solution [35], the central singularity appears at $t=\left(6 \pi \rho_{c, i}\right)^{-1 / 2}$, while the surface is swallowed by an event horizon at $t=\left(6 \pi \bar{\rho}_{i}\right)^{-1 / 2}-4 M / 3$, where $t$ is the orthogonal time coordinate and $\rho_{c, i}$ and $\bar{\rho}_{i}$ are the initial central density and average density of the dust cloud at $t=0$, respectively. It is obvious that the former can be earlier than the latter for the initial data with certain central concentration.

[55] The similar results were obtained for linearized gravitational waves during the spherically symmetric homogeneous dust collapse [22] and for scalar waves during the neutron star collapse in the fully nonlinear spherically symmetric Einsteinfluid-Klein-Gordon system [43]. 


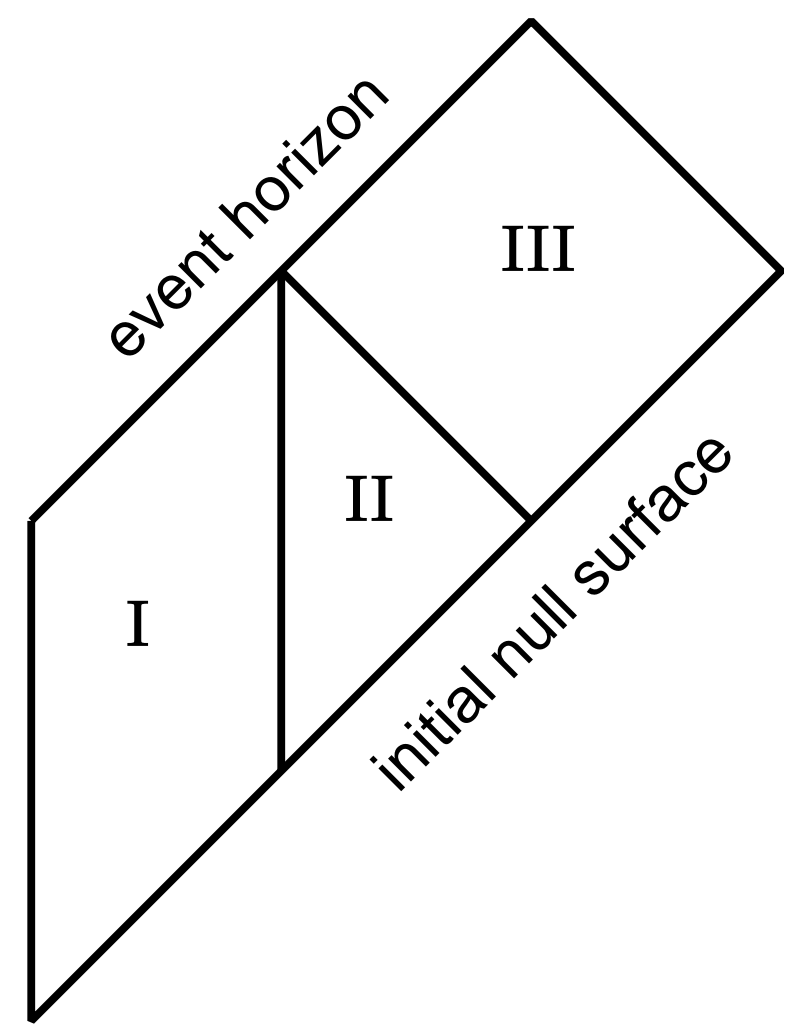

FIG. 1: Spacetime structure of spherically symmetric black hole formation is depicted. Region I denotes the stellar interior, region II denotes the intermediate exterior region from which we can emit an ingoing light ray which hits the stellar surface before the horizon formation, and region III denotes the far exterior region. The boundary between regions I and II is the stellar surface. 


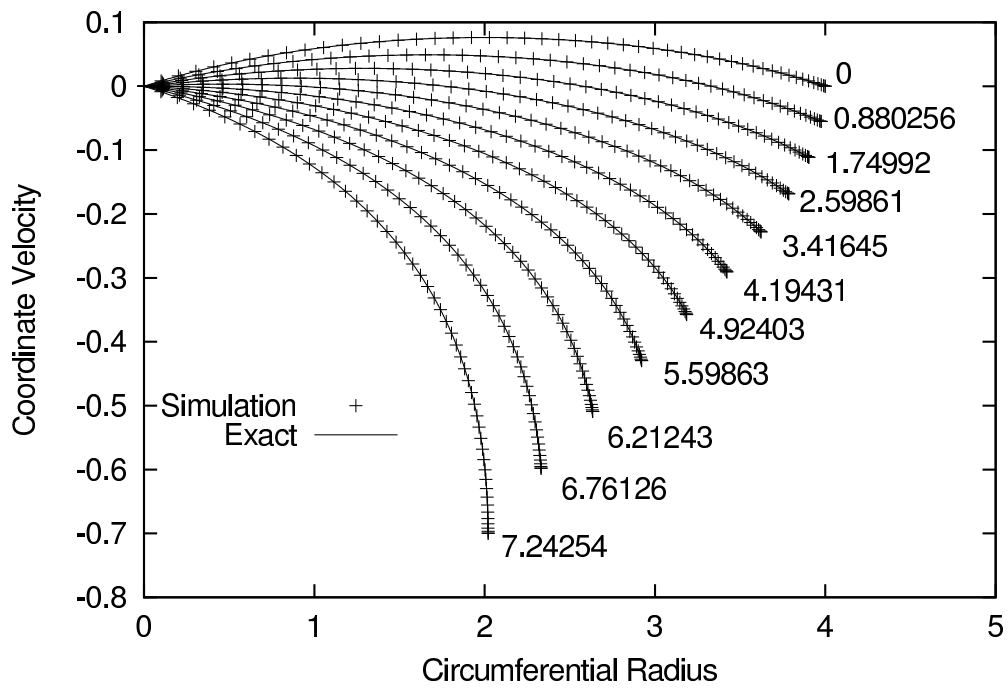

FIG. 2: Snapshots of the velocity profile in the numerical simulation of homogeneous dust collapse with the initial radius $R=4 M$. Crosses and solid curves denote the numerical and exact solutions. The initial data set is put on the initial outgoing null cone on which the dust surface is momentarily static. The vertical and horizontal axes are the coordinate velocity $U=e^{-\psi} R_{, u}$ and the circumferential radius $R$, respectively. The attached labels denote the proper time $\tau_{\mathrm{s}}$ of a comoving observer at the stellar surface. All the quantities are shown in units of $M=1$. 
(a)

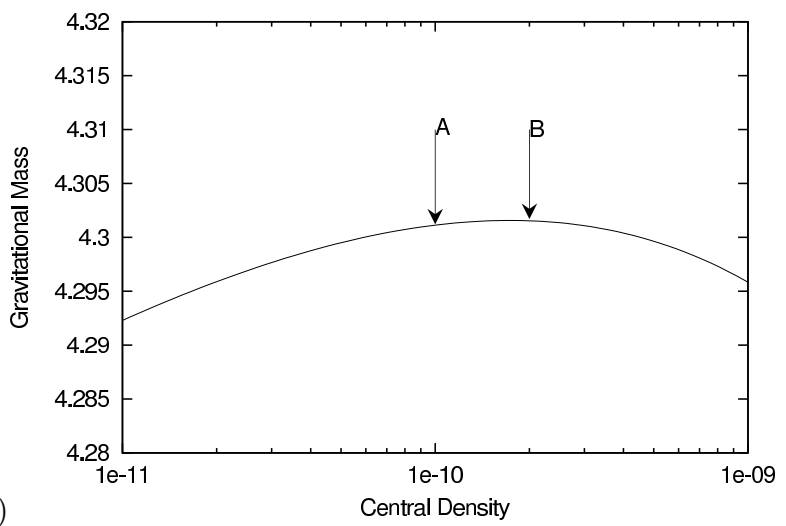

(b)

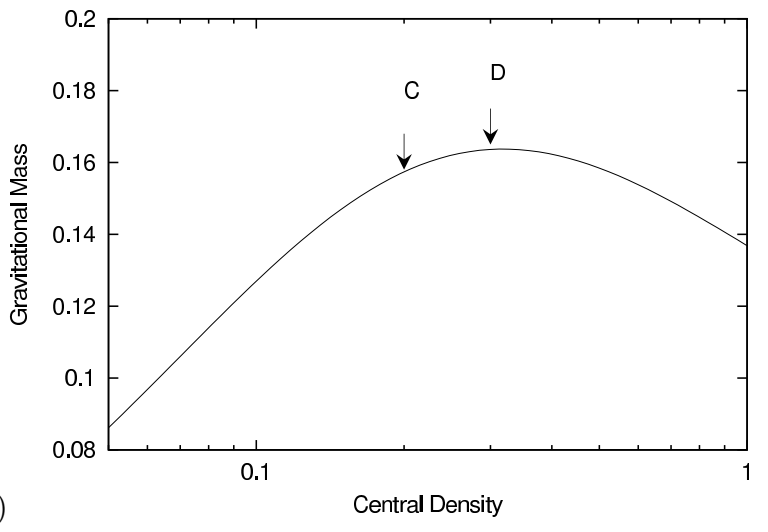

FIG. 3: Gravitational mass as a function of central density of spherical equilibrium stars with polytropic equations of state $P=K n^{\Gamma_{\mathrm{a}}}$, where (a) $\Gamma_{\mathrm{a}}=(4 / 3)+0.00142$ and (b) $\Gamma_{\mathrm{a}}=2$. Models A and C are stable while models B and D are marginally stable against gravitational collapse. The numerical values are shown in units of $c=G=K=1$.
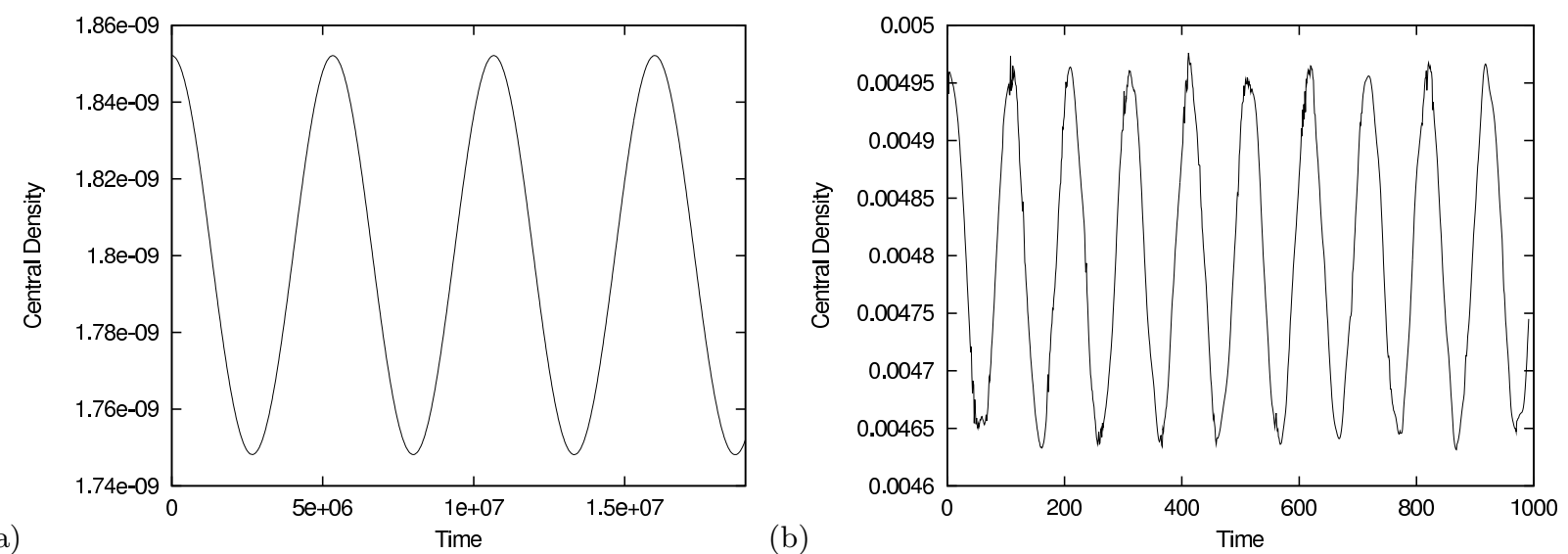

FIG. 4: Evolutions of the central density (a) for model A with $\Gamma_{\mathrm{a}}=(4 / 3)+0.00142$ and (b) for model $\mathrm{C}$ with $\Gamma_{\mathrm{a}}=2$. The horizontal axis is the time $\bar{u}$ for an observer at infinity. The internal energy $e$ is initially increased uniformly by $1 \%$ both for (a) and for (b) from the equilibrium configuration. All the quantities are shown in units of $M=1$. 
(a)

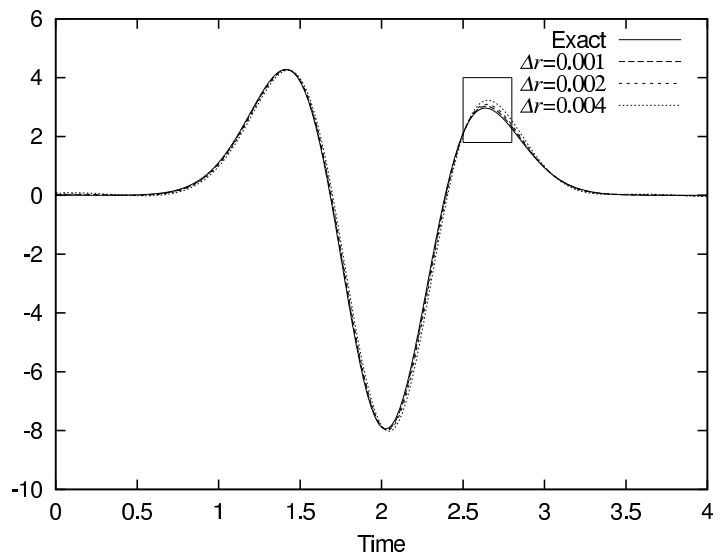

(b)

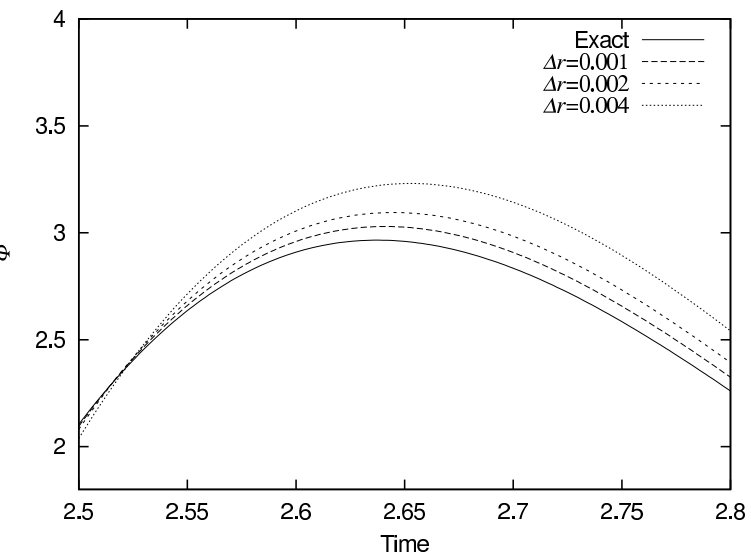

FIG. 5: Linear gravitational waveforms for $l=2$ in the Minkowski spacetime extracted at $r=5$. The initial data set is provided on the initial null surface $t=r$. Three levels of the grid resolution as $\Delta r=\Delta u=\Delta v=0.001,0.002$, and 0.004 are adopted. The solid line denotes the exact solution. (b) is the magnification of the region encompassed by the square in (a).

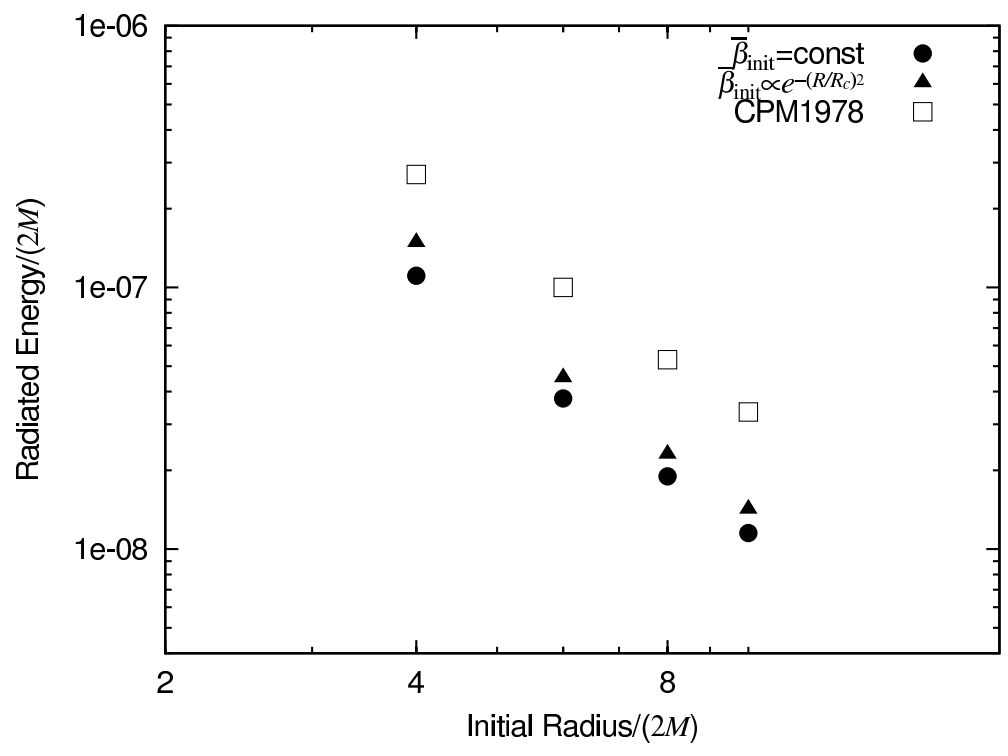

FIG. 6: Total radiated energy of gravitational waves for $l=2$ from homogeneous dust collapse for different initial (maximally expanded) radii. The initial moment $q$ is normalized so that $q=2 M$. We set the momentarily static initial data on the initial null slice. The results for two kinds of initial distribution for matter perturbation $\bar{\beta}_{\text {init }}$ are plotted. For the case of $\bar{\beta}_{\text {init }} \propto \exp \left[-\left(R / R_{c}\right)^{2}\right]$, the radius $R_{c}$ is chosen to be one-third of the initial radius of the dust ball. The present results are compared with those of Cunningham, Price, and Moncrief [22] (CPM1978), in which the initial data sets were set on the spacelike surface of maximum expansion. 
(a)

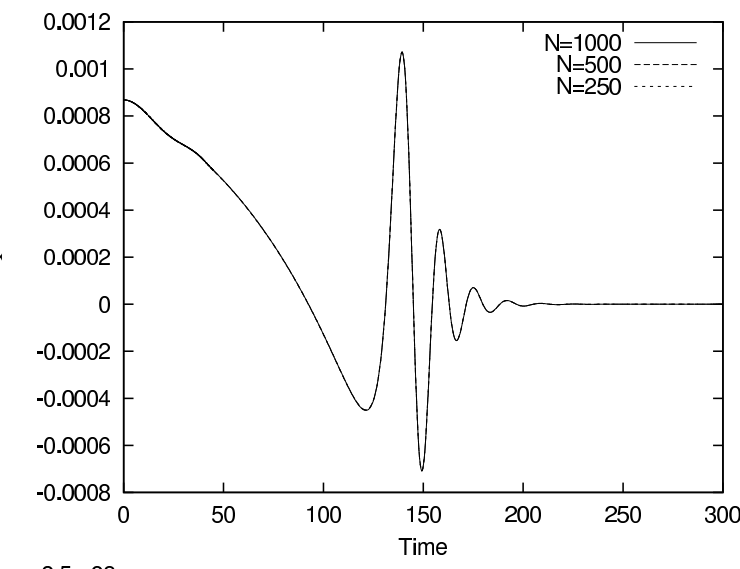

(c)

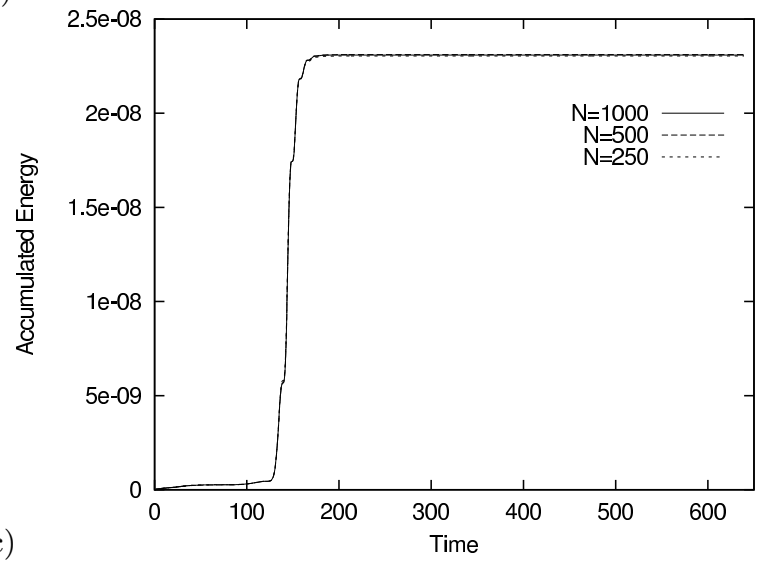

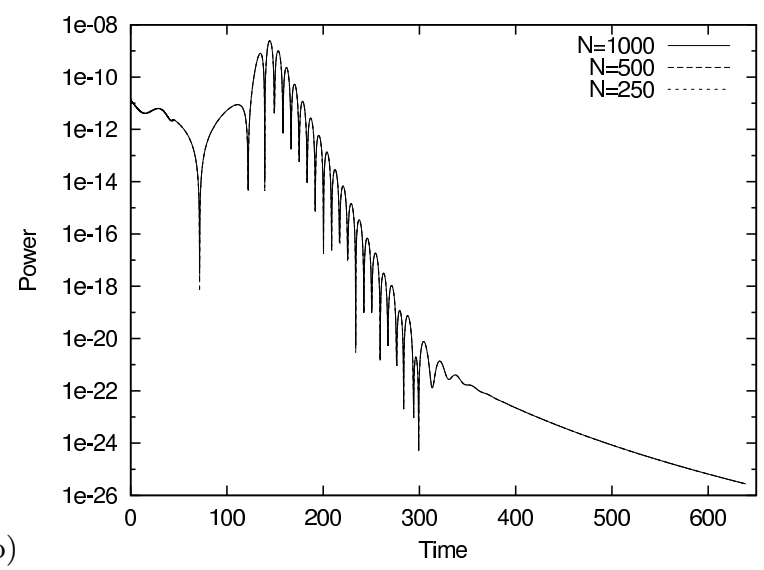

(b)

FIG. 7: (a) Waveform, (b) luminosity, and (c) accumulated energy of gravitational waves for $l=2$ radiated from homogeneous dust collapse with the initial radius $R=20 M$. These are estimated at $R=40 M$ and normalized so that $q=2 M$. The horizontal axis is the observer time $\bar{u}$. We set the momentarily static initial data on the initial null surface with the matter perturbation $\bar{\beta}_{\text {init }}=$ const. The solid, long-dashed, and dashed lines denote the results for $N=1000,500$, and 250, respectively, where $N$ is the number of spatial grid points inside the dust ball. The matching is done at the outermost mass shell. All the quantities are shown in unit of $M=1$. The long-dashed and dashed lines are almost indistinguishable because they lie on top of the solid line. 
(a)

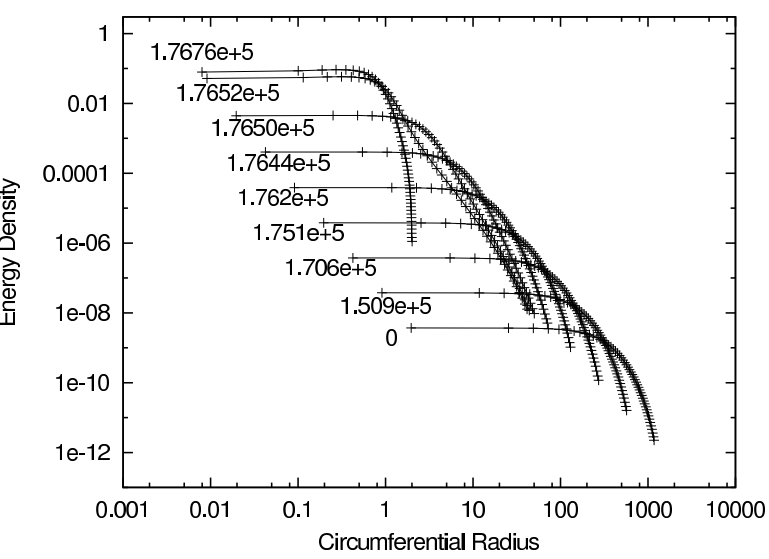

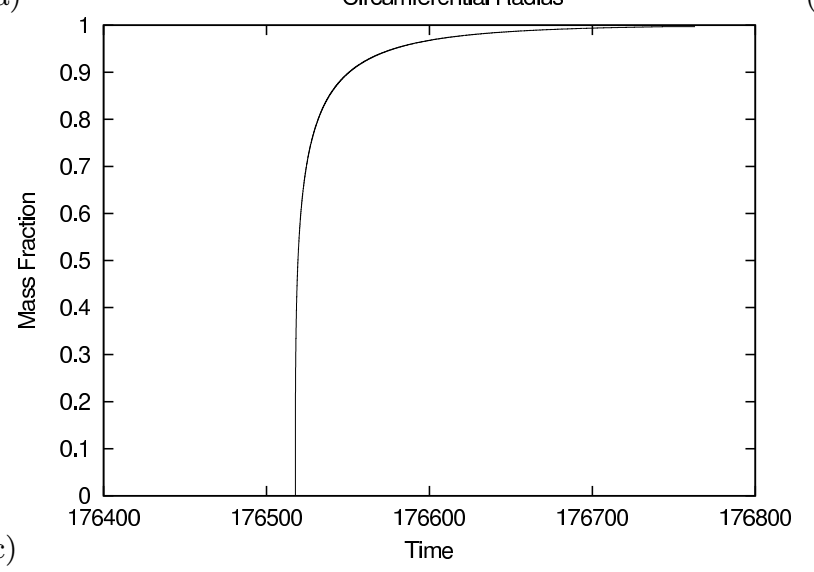

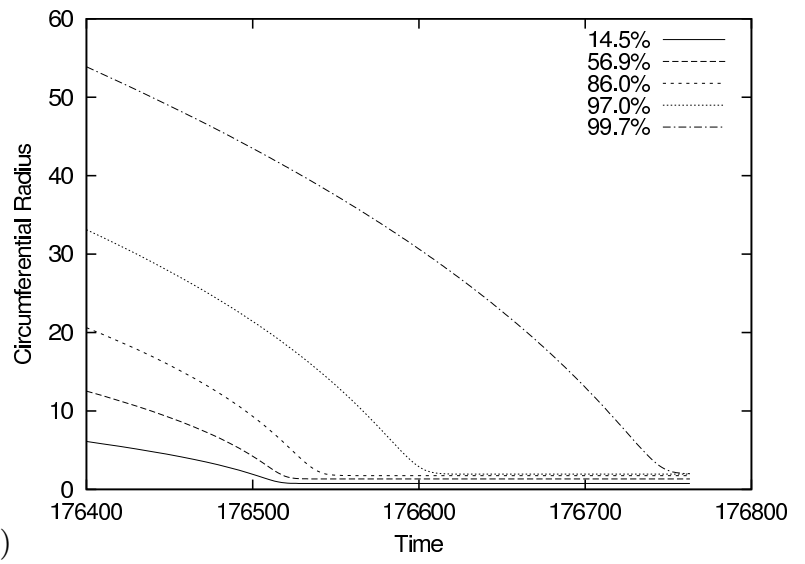

FIG. 8: (a) Snapshots of the density profile, (b) the trajectories of mass shells, and (c) the mass fraction contained in a high-redshift region are plotted for the gravitational collapse of model B with $\Gamma_{\mathrm{a}}=(4 / 3)+0.00142$. We extract $1 \%$ of the internal energy from the equilibrium configuration to induce the collapse. In (a) the label for each curve denotes the observer time $\bar{u}$. In (b) the vertical and horizontal axes denote the circumferential radius and observer time, respectively. The labels denote the mass fractions enclosed by mass shells. In (c) the high-redshift region is defined as the one in which the lapse function $\alpha$ is less than 0.1. We deal with the mass shell which encloses $99.7 \%$ of the total mass as the stellar surface. All the quantities are shown in units of $M=1$. 
(a)
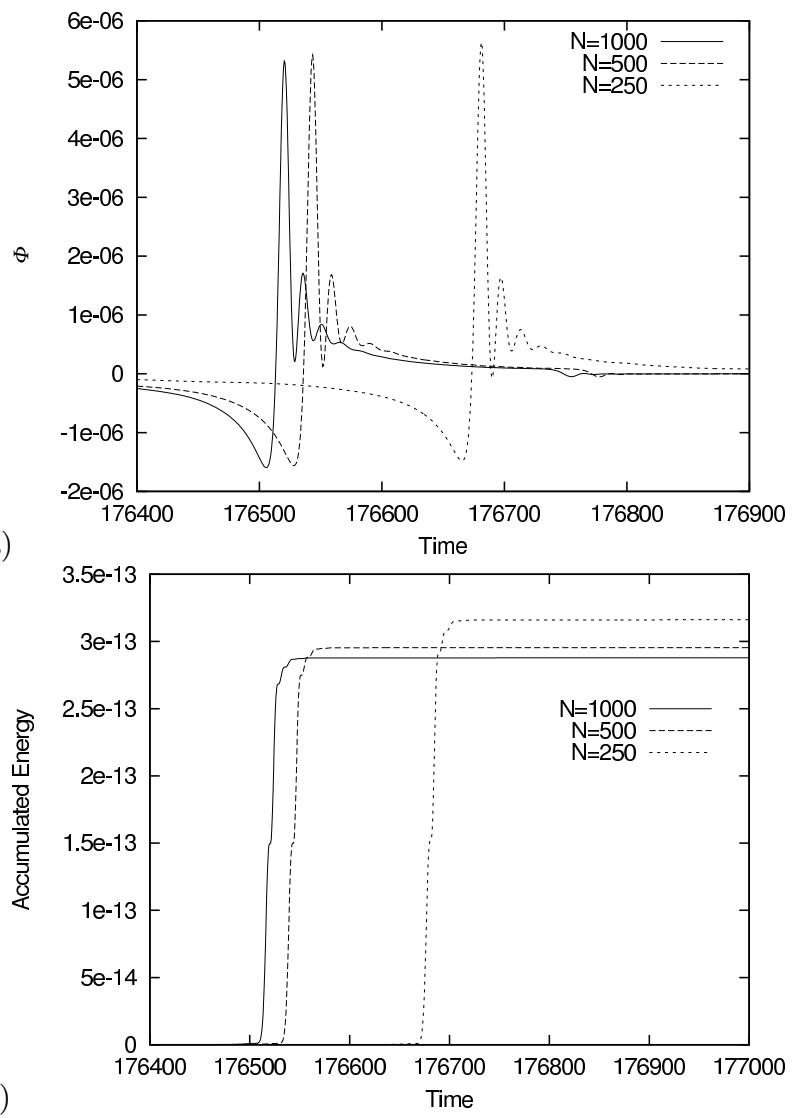

(c)

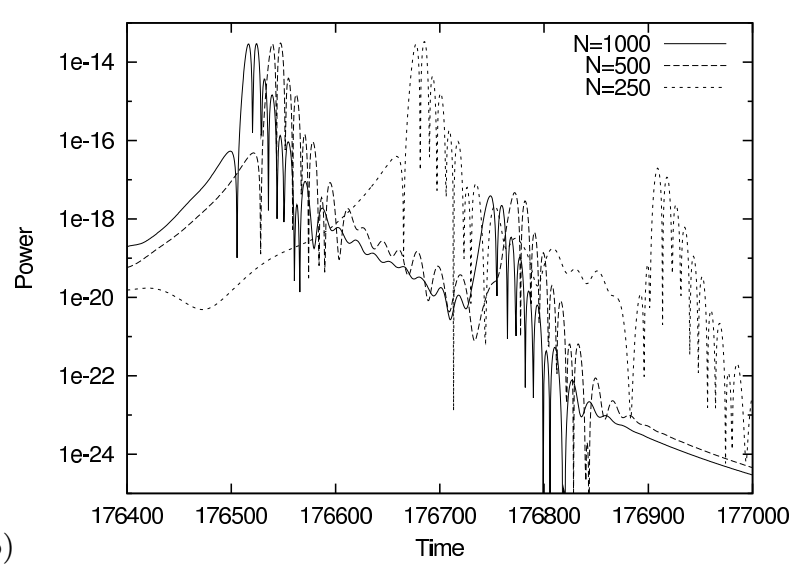

(b)

FIG. 9: (a) Waveform, (b) luminosity and (c) accumulated energy of gravitational waves for $l=2$ radiated from the collapse of model B with $\Gamma_{\mathrm{a}}=(4 / 3)+0.00142$. Gravitational waves are extracted at $R=2000 M$. The initial matter perturbation is chosen so that $\bar{\beta}_{\text {init }}=$ const. The momentarily static initial perturbation is given. The amplitude of the perturbation is normalized so that $q=2 M$. The solid, long-dashed, and dashed lines denote the results for $N=1000,500$, and 250, respectively, where $N$ is the number of spatial grid points inside the star. The matching is done on the mass shell which encloses $99.7 \%$ of the total mass. All the quantities are shown in units of $M=1$. 
(a)

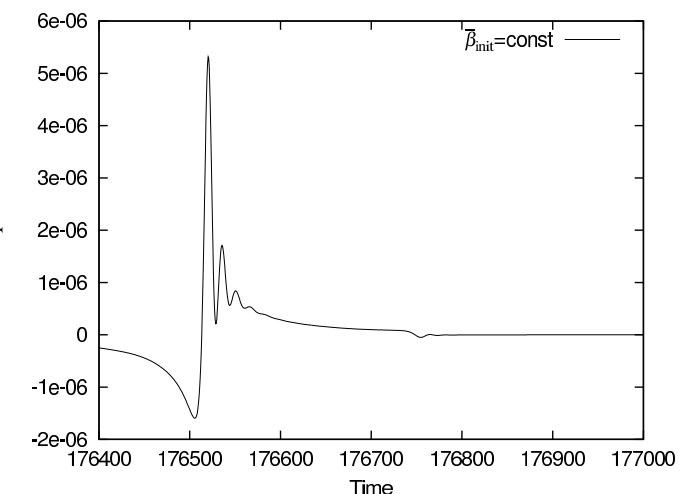

(c)

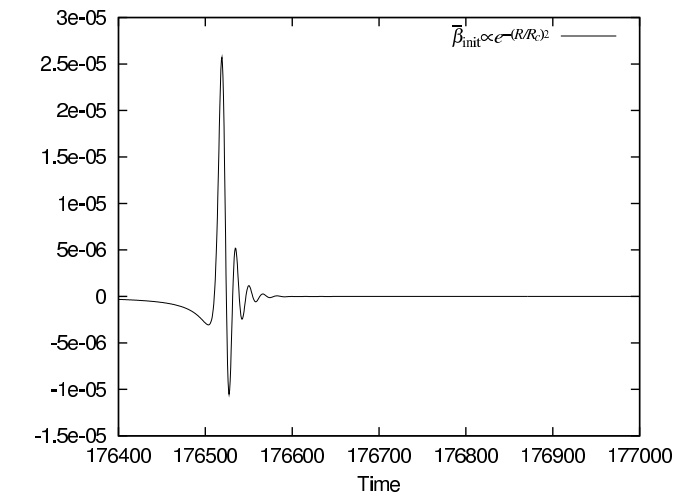

(e)
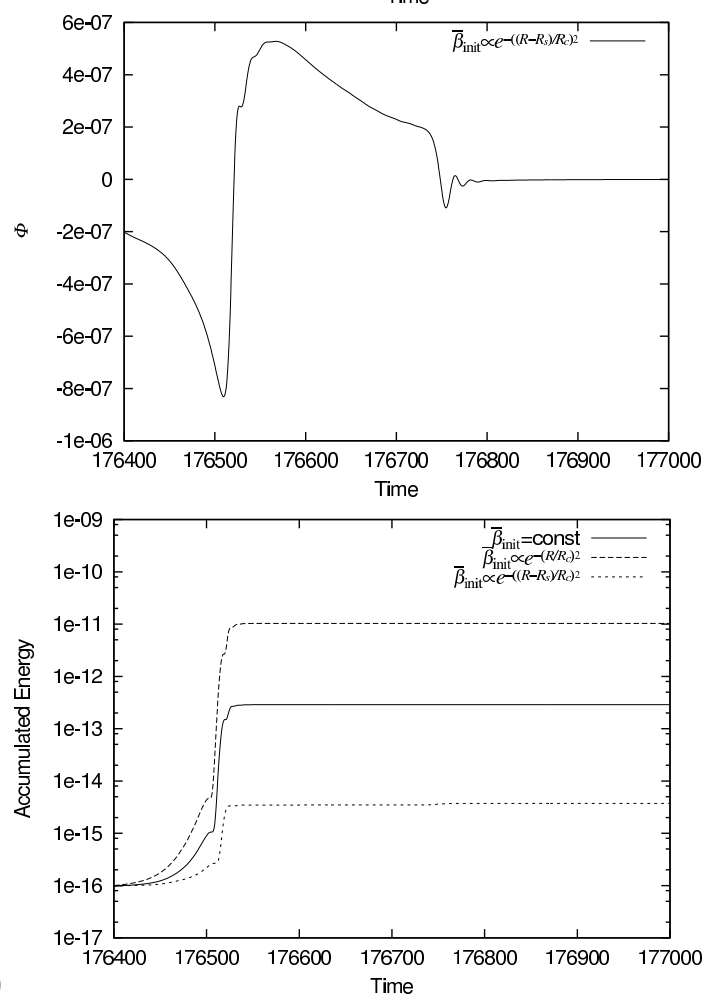

(b)
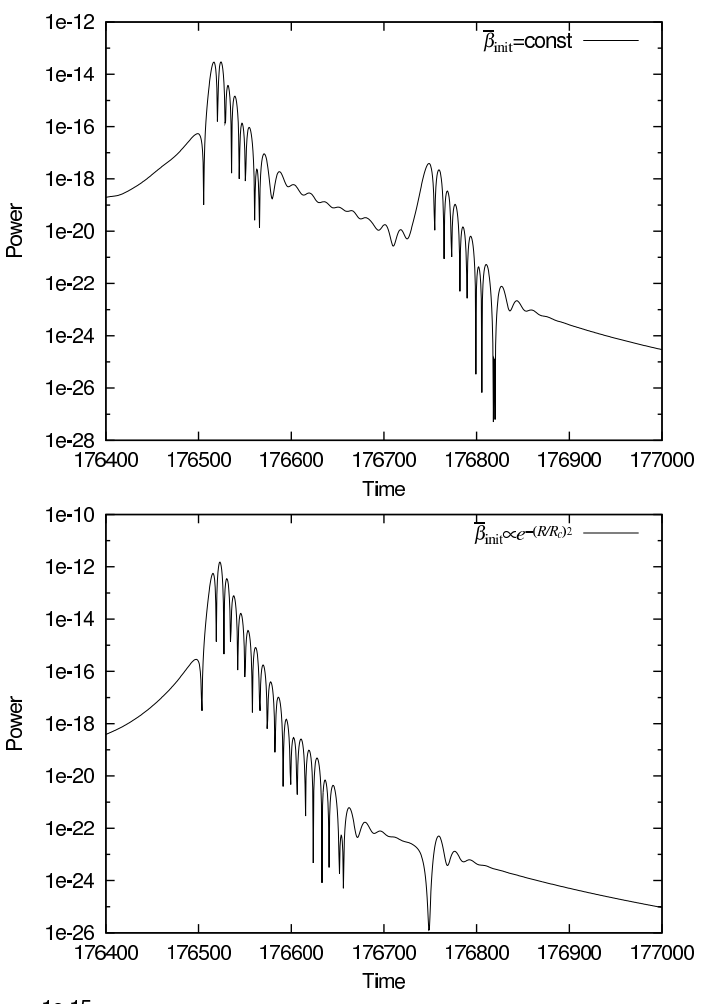

(d)
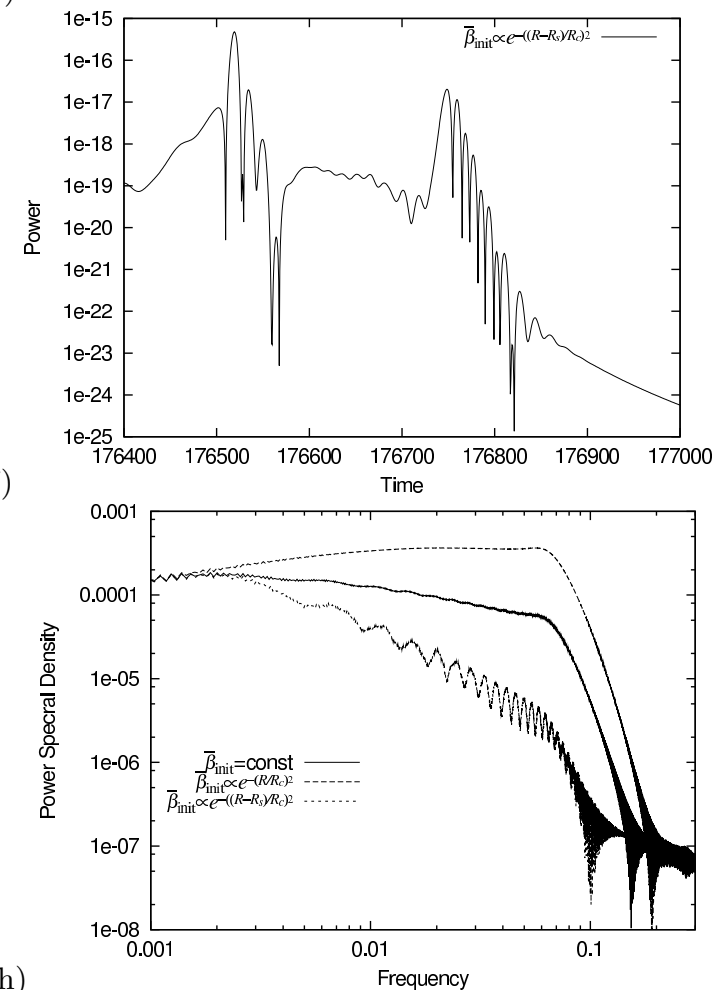

FIG. 10: (a), (c), and (e) Waveform, (b), (d), and (f) luminosity, (g) accumulated energy, and (h) the one-sided power spectral density of gravitational waves for $l=2$ radiated from the collapse of model B with $\Gamma_{\mathrm{a}}=(4 / 3)+0.00142$. Gravitational waves are extracted at $R=2000 M$. The momentarily static initial perturbation is given. The amplitude of the perturbation is normalized so that $q=2 M$. In $(\mathrm{g})$ and $(\mathrm{h})$, the solid, long-dashed, and dashed lines denote the results for $\bar{\beta}_{\text {init }}=$ const, $\exp \left[-\left(R / R_{\mathrm{c}}\right)^{2}\right]$, and $\exp \left\{-\left[\left(R-R_{\mathrm{s}}\right) / R_{\mathrm{c}}\right]^{2}\right\}$, respectively, where $\bar{\beta}_{\text {init }}$ is the initial distribution of the renormalized matter perturbation and $R_{\mathrm{c}}$ is chosen to be $R_{\mathrm{c}}=R_{\mathrm{s}} / 3$. For waveforms and luminosities, we display the results for each case in each figure for clarity. Note that the vertical axis of $(\mathrm{g})$ is in logarithmic scale. The matching is done on the mass shell which encloses $99.7 \%$ of the total mass. All the quantities are shown in units of $M=1$. 
(a)
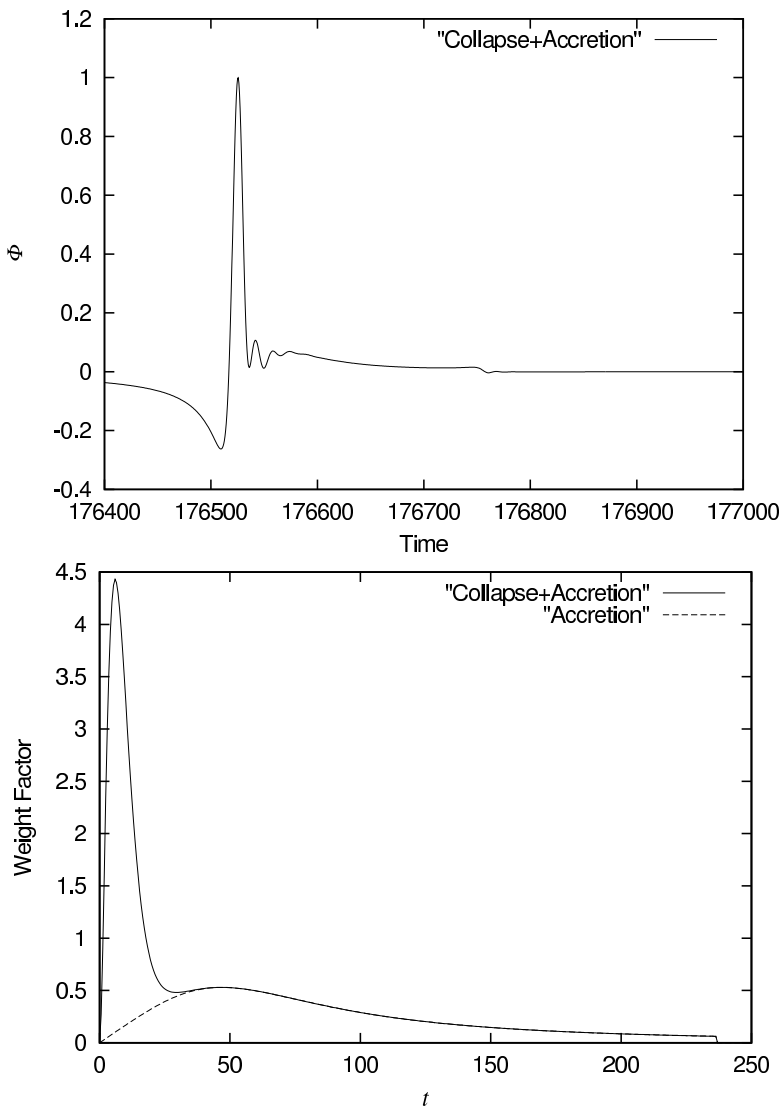

(c) (b)

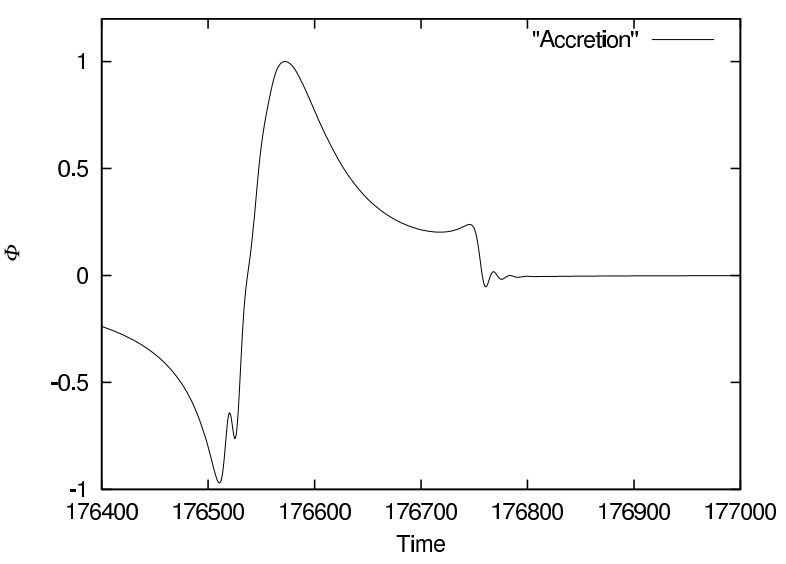

FIG. 11: Waveforms generated by superimposing the waveform obtained for case (2). The shape of weight factors are displayed in (c). (a) and (b) show the waveforms for the weight factor labelled "Collapse+Accretion" and for that labelled "Accretion." The waveforms are normalized so that the maximum amplitude is unity. See text for details. 
(a)
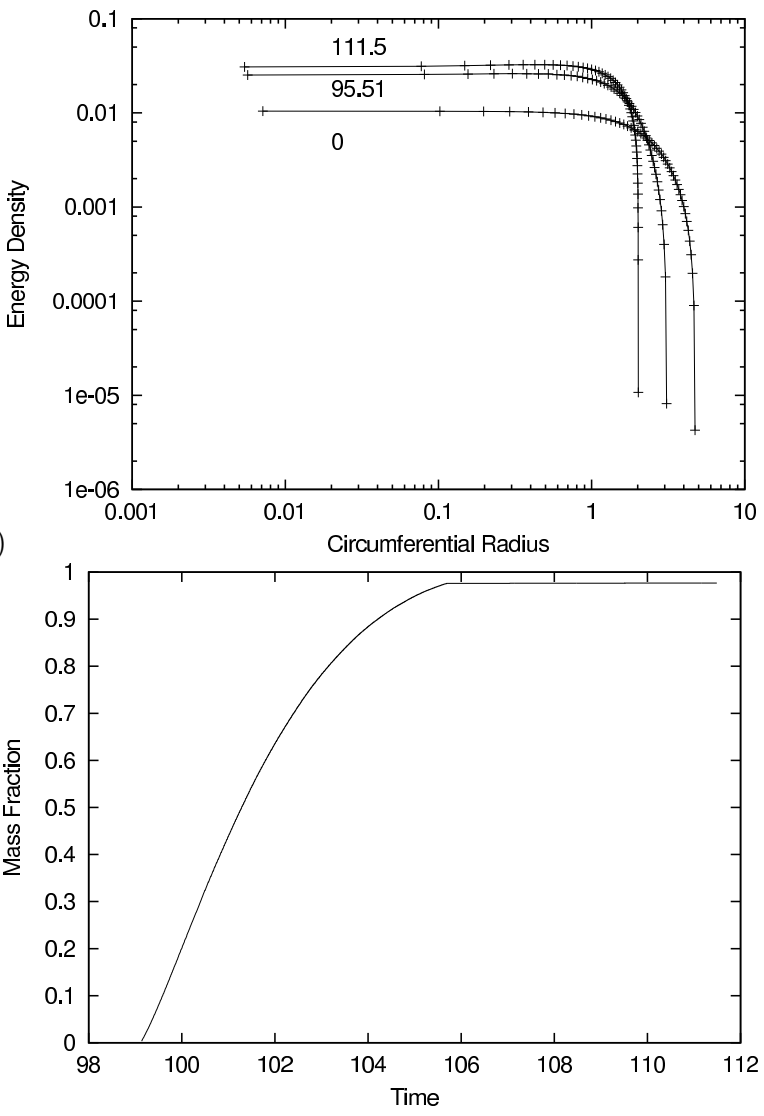

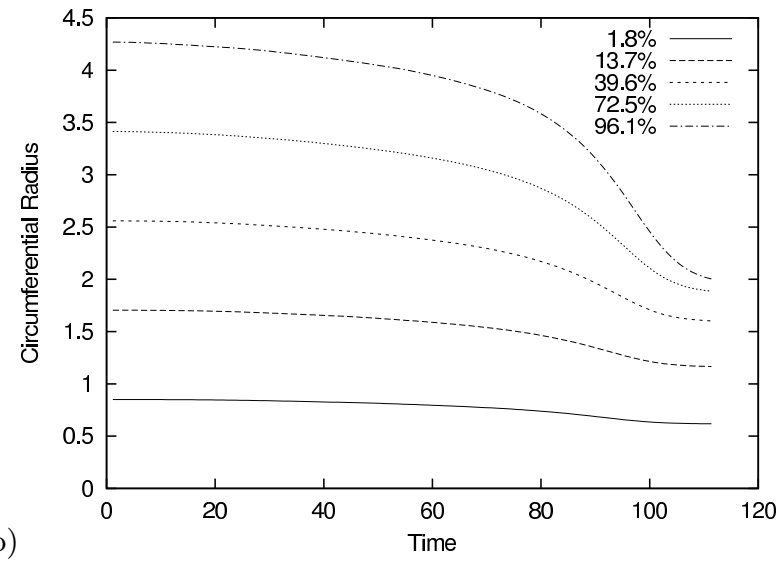

(b)

(c)

FIG. 12: Same as Fig. 8 but for the collapse of model D with $\Gamma_{\mathrm{a}}=2$. We deal with the mass shell which encloses $96.1 \%$ of the total mass as the stellar surface. 
(a)
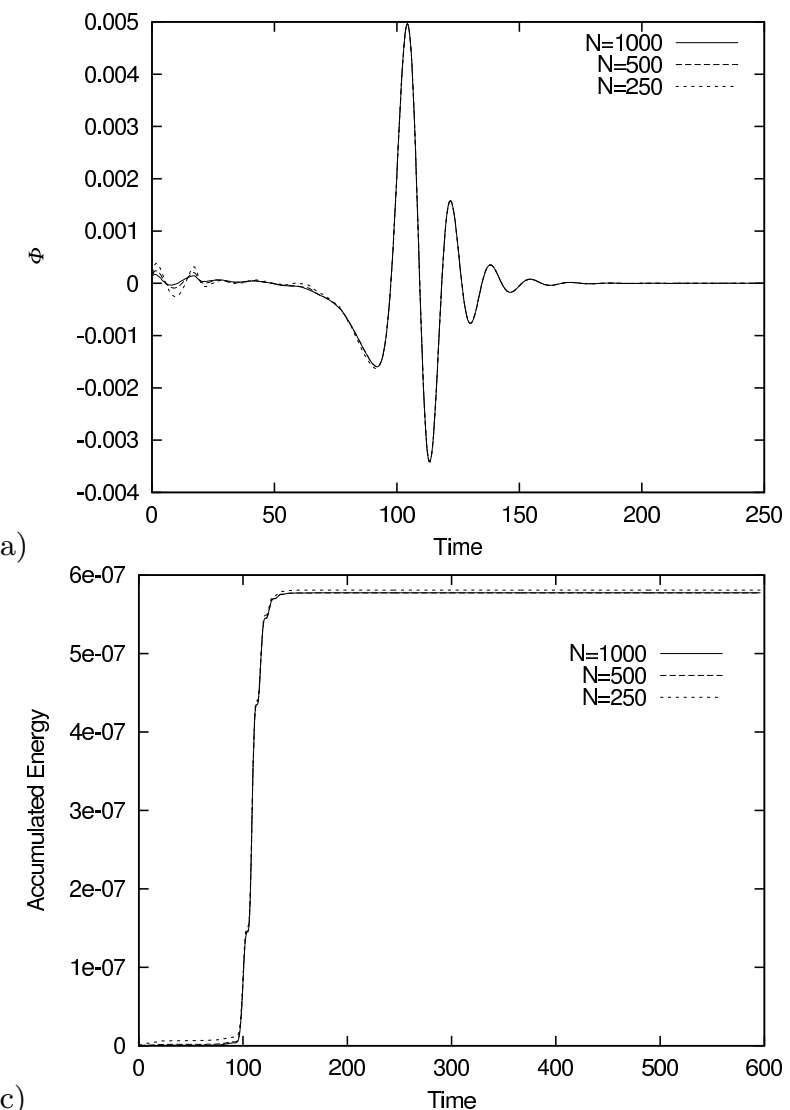

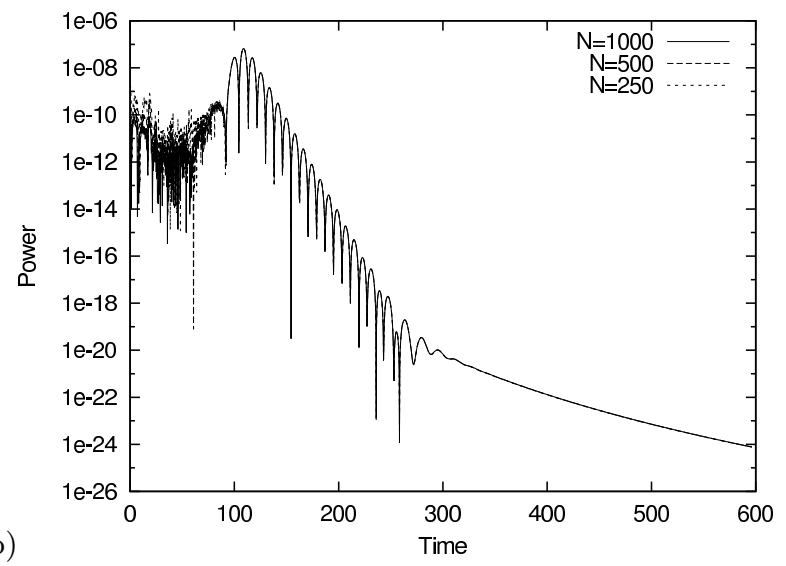

(b)

(c)

FIG. 13: Same as Fig. 9 but for the collapse of model D with $\Gamma_{\mathrm{a}}=2$. Gravitational waves are extracted at $R=100 M$. The matching is done on the mass shell which encloses $96.1 \%$ of the total mass. All the quantities are shown in units of $M=1$. 
(a)
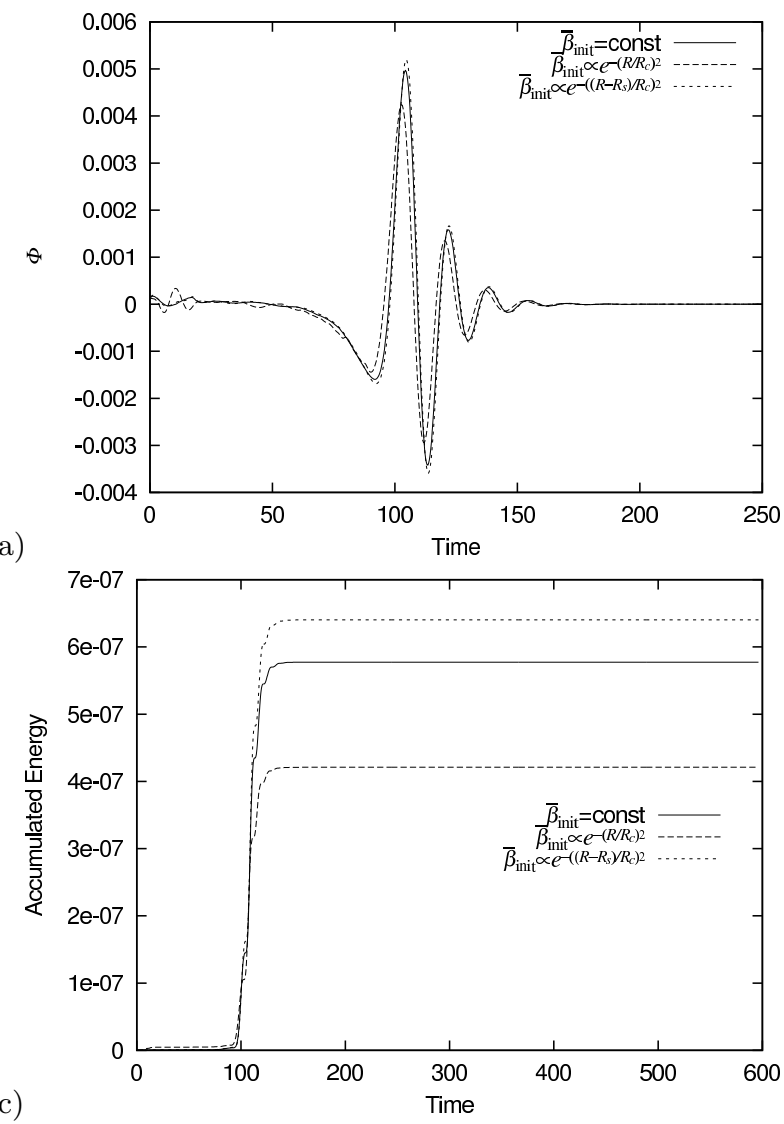

(b)

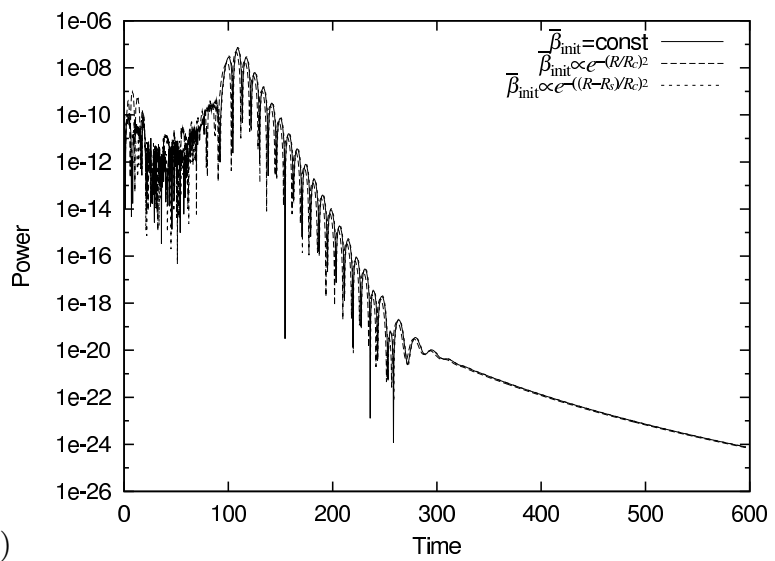

(d)

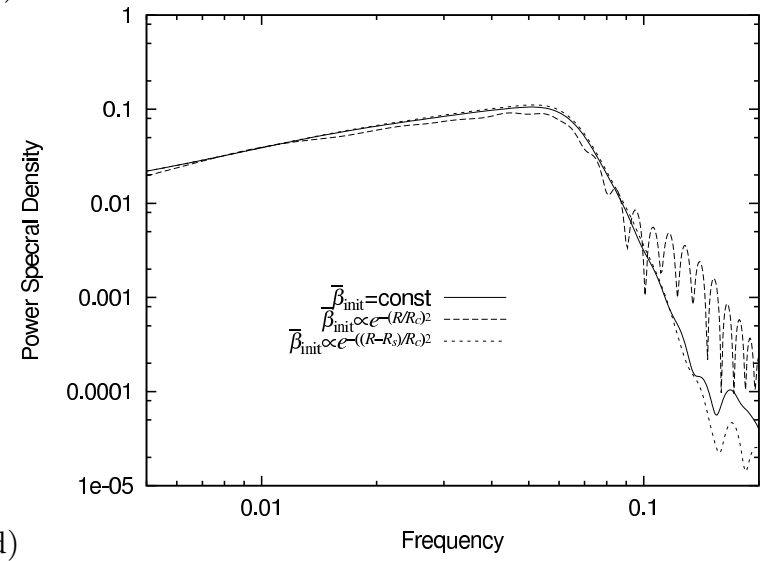

FIG. 14: (a) Waveform, (b) luminosity, (c) accumulated energy, and (d) one-sided power spectral density of gravitational waves for $l=2$ radiated from the collapse of model $\mathrm{D}$ with $\Gamma_{\mathrm{a}}=2$. Gravitational waves are extracted at $R=100 M$. The momentarily static initial perturbation is given. The amplitude of the perturbation is normalized so that $q=2 M$. The solid, long-dashed and dashed lines denote the results for $\bar{\beta}_{\text {init }}=$ const, $\exp \left[-\left(R / R_{\mathrm{c}}\right)^{2}\right]$, and $\exp \left\{-\left[\left(R-R_{\mathrm{s}}\right) / R_{\mathrm{c}}\right]^{2}\right\}$, respectively, where $\bar{\beta}_{\text {init }}$ is the initial distribution of the renormalized matter perturbation and $R_{\mathrm{c}}$ is chosen to be $R_{\mathrm{c}}=R_{\mathrm{s}} / 3$. The matching is done on the mass shell which encloses $96.1 \%$ of the total mass. All the quantities are shown in units of $M=1$. 